\title{
Nascent polypeptide within the exit tunnel ensures continuous translation elongation by stabilizing the translating ribosome
}

\author{
Yuhei Chadani $^{1 *}$, Nobuyuki Sugata ${ }^{2}$, Tatsuya Niwa ${ }^{1,2}$, Yosuke Ito $^{2}$, Shintaro Iwasaki ${ }^{3}$ \\ and Hideki Taguchi ${ }^{1,2 *}$
}

${ }^{1}$ Cell Biology Center, Institute of Innovative Research, Tokyo Institute of Technology, Yokohama, Japan.

${ }^{2}$ School of Life Science and Technology, Tokyo Institute of Technology, Yokohama, 226-8503, Japan.

3. RNA Systems Biochemistry Laboratory, RIKEN Cluster for Pioneering Research, Saitama, Japan.

*Correspondence to: chadani.y.aa@m.titech.ac.jp, taguchi@bio.titech.ac.jp. 


\section{Summary}

Continuous translation elongation, irrespective of amino acid sequences, is a prerequisite for living organisms to produce their proteomes. However, the risk of elongation abortion is concealed within nascent polypeptide products. Negatively charged sequences with occasional intermittent prolines, termed intrinsic ribosome destabilization (IRD) sequences, destabilizes the translating ribosomal complex. Thus, some nascent chain sequences lead to premature translation cessation. Here, we show that the risk of IRD is maximal at the $\mathrm{N}$-terminal regions of proteins encoded by dozens of Escherichia coli genes. In contrast, most potential IRD sequences in the middle of open reading frames remain cryptic. We found two elements in nascent chains that counteract IRD: the nascent polypeptide itself that spans the exit tunnel and its bulky amino acid residues that occupy the tunnel entrance region. Thus, nascent polypeptide products have a built-in ability to ensure elongation continuity by serving as a bridge and thus by protecting the large and small ribosomal subunits from dissociation. 
Introduction

The ribosome is the central player in translation, at the heart of the central dogma,

3 in all organisms. Accurate and continuous peptide bond formation by the ribosome ensures the uniformity and quality of cellular proteins. Otherwise, aberrant polypeptides would lead to undesired outcomes such as impaired or dysregulated protein functions and aggregation, which perturb cellular homeostasis. Thus, living organisms have multilayered surveillance systems over ribosomes and translation, including proofreading of codon-anticodon recognition (Ieong et al., 2016; Noel and Whitford, 2016; Rodnina and Wintermeyer, 2001), quality control pathways to sense unusual translation reactions, and degradation of premature products (Buskirk and Green, 2017; Inada, 2017; Keiler, 2008).

Translation is initiated when the small ribosomal subunit (the $30 \mathrm{~S}$ in prokaryotes) recognizes the start codon within mRNA, and this is followed by the recruitment of the initiator aminoacyl-tRNA and the association of the large ribosomal subunit (50S in prokaryotes). In the decoding process, aminoacyl-tRNAs are delivered as ternary complexes with EF-Tu into the A-site of the ribosome complex. After the decoding, EF-Tu dissociates from the ribosome. The peptidyl transferase center (PTC) of the ribosome catalyzes peptide bond formation between the P-site and the A-site amino acids. The ribosomal subunits then dynamically rotate to translocate the peptidyl-tRNA through the $\mathrm{A} / \mathrm{P}$ hybrid state to the $\mathrm{P} / \mathrm{P}$ classic state, with the concomitant one-codon advancement of the ribosome along the mRNA. The complex is now ready to proceed to the next cycle of peptidyl-transfer for continuous translation elongation. The nascent peptide grows until a release factor recognizes the stop codons within the A-site and then cleaves the peptidyl-tRNA ester bond. Once the synthesized polypeptide is released, the ribosome is split into two subunits by the cooperative actions of the ribosome recycling factor and EF-G to recycle them for the next round of translation (Choi et al., 2015; Rodnina, 2016, 2018; Rodnina and Wintermeyer, 2016).

Structural studies have invariably shown that the ribosomes possess a tunnel structure (exit tunnel) that accommodates 30-40 amino acid-long segments of tRNAlinked nascent polypeptides (Jenni and Ban, 2003; Voss et al., 2006). During the elongation process, each amino acid residue of a growing nascent polypeptide travels from the PTC through the exit tunnel of the large ribosomal subunit to the extra-ribosomal space (Wilson and Beckmann, 2011). The tunnel is composed of the large ribosomal RNA and the tips of ribosomal proteins, such as uL4, uL22, and uL23 (Nissen et al., 2000). In contrast to the initial suggestion that the tunnel interior is inert in terms of interactions 
37 nascent polypeptide chains, thus contributing to biological regulation (Chiba et al., 2009;

38 Gong and Yanofsky, 2002; Ishii et al., 2015; Nakatogawa and Ito, 2002; Onouchi et al., 39 2005; Yanagitani et al., 2011, Ito and Chiba, 2013). Cryo-EM structures of ribosomes 40 stalled by ribosome arresting peptides (RAPs) have revealed a range of interactions 41 between the nascent chains and the ribosomal interior components at the PTC and/or the 42 exit tunnel, such as the uL22/uL4 constriction site (Bhushan et al., 2011; Seidelt et al., 43 2009; Shanmuganathan et al., 2019; Sohmen et al., 2015; Su et al., 2017). Recent analyses using ribosome profiling (Dao Duc and Song, 2018; Han et al., 2020; Requião et al., 2016; Sabi and Tuller, 2015) and direct detection of accumulating peptidyl-tRNAs (Chadani et al., 2016) have suggested the widespread occurrence of translation pausing, to which nascent polypeptides may contribute. Thus, nascent polypeptides are not simple intermediates in translation; in contrast, they actively regulate the ribosomal functions.

Our recent study demonstrated that nascent polypeptides within the ribosome can induce intrinsic ribosome destabilization (IRD), leading to a premature cessation of translation (Chadani et al., 2017). When the ribosome translates a sequence with consecutive negatively-charged residues or intermittent with prolines (DE/P motif), its subunit structure becomes destabilized. Such nascent polypeptides become susceptible to peptidyl-tRNA hydrolase (Pth), which otherwise cannot access a substrate sequestered within the intact ribosome complex (Das and Varshney, 2006; Sharma et al., 2014). Thus, translation is aborted stochastically within the DE/P motif. Deleting bL31, which bridges the large and small subunits, destabilizes the 70S structure and enhances IRD. Thus, specific amino acid sequences within a nascent polypeptide can compromise the ribosome's structural integrity and translation continuity. Remarkably, IRD contributes to gene regulation. MgtL, the leader peptide of MgtA (Gall et al., 2016; Park et al., 2010; Zhao et al., 2011), an $\mathrm{Mg}^{2+}$ transporter, contains a potential IRD-inducing motif, EPDP, at its N-terminus. The IRD in MgtL is exaggerated under ribosome-destabilizing conditions, including lowered $\mathrm{Mg}^{2+}$ concentrations, triggering the MgtA-upregulating genetic scheme. Whereas MgtL provides an example of programmed IRD in cells, our knowledge about the roles of nascent peptides in regulating the stability, in either direction, of the ribosomes in action remains incomplete.

Here, we report our proteome-wide profiling of the potential IRD-inducing sequences (hereafter, called "IRD sequences") in E. coli and our analyses of how this organism maintains robust translation continuity by overcoming IRD. We identified dozens of N-terminal IRD sequences that cause significant premature translation cessation. By contrast, the IRD-like sequences in the open reading frames' internal regions are silent, raising the possibility that a preceding ( $\mathrm{N}$-terminal) nascent 
polypeptide segment residing in the exit tunnel antagonizes IRD. We found two IRDcounteracting elements in nascent polypeptides: their lengths that occupy the tunnel and the bulkiness of the amino acid side chains in the PTC-adjacent region. The "length" and "bulkiness" of nascent chains affect the genome-wide amino acid distribution in ORFs to minimize the risk of nonproductive translation discontinuation. These results shed new light on the pivotal roles played by nascent chains in the ribosomal exit tunnel in ensuring the efficient synthesis, without delay, of every possible peptide with any sequence context required by living organisms.

\section{Results}

\section{N-terminal DE/P-rich sequences in $E$. coli ORFs induce IRD.}

We previously showed that the "EPDP" sequence following the initiator methionine of E. coli MgtL destabilizes the translating $70 \mathrm{~S}$ ribosome from within to prematurely abort translation, which is harnessed as an environmental sensing strategy (Chadani et al., 2017). As the translation of MgtL aborts within the first six amino acid residues (MEPDPT) of MgtL, other proteins with MgtL-like $\mathrm{N}$-terminal sequences could also induce premature translation abortion. We searched the 4,187 E. coli ORFs for those with sequences enriched in $\mathrm{D}, \mathrm{E}$ and $\mathrm{P}$ in the six amino acid windows following the $\mathrm{N}$ terminal methionine, and found ten genes as candidates. To determine whether these DE/P-enriched N-terminal sequences indeed cause IRD sequence-dependent translation discontinuation (hereafter referred to as "IRD"), we then fused the N-terminal ten amino acid sequences ( $\mathrm{Nt10}$ ) of the candidate genes to LacZ (Figure 1A) and measured the LacZ activities. As controls, we also constructed their "no DE/P" variants, in which the Nterminal D, E, and P residues were replaced by $\mathrm{N}$ (Asn), Q (Gln), and A (Ala), respectively.

We normalized the enzyme activity of each construct by that of the respective no $\mathrm{DE} / \mathrm{P}$ variant, to obtain the quantitative indications, termed the translation continuation (T.C.) index, in which $100 \%$ and $0 \%$ values represent no IRD and complete DE/Pdependent translation discontinuation, respectively. Out of the eight candidates with sufficient expression levels, seven showed translation continuation indices of less than 50

104 (Figure 1B). These translation anomalies at the N-terminal regions were independent of the $5^{\prime}$ untranslated regions (UTRs) (Figure S1A and S1B). In addition, a low $\mathrm{Mg}^{2+}$

106 concentration and the deletion of bL31, conditions that destabilize the 70S ribosome complex, enhanced the DE/P-dependent translation attenuation (Figure S1C and S1D). 
108 These results suggest that IRD occurred during the translation of the N-terminal DE/Prich regions of these ORFs.

We next studied the translation of the Nt10 constructs in the PURE system, a reconstituted in vitro translation system (Shimizu et al., 2001), to recapitulate the

112 translation abortion using defined translation components. Nt10 constructs with a

113 shortened lacZ fragment were translated by the PURE system in the presence of ${ }^{35} \mathrm{~S}$ -

114 methionine, and the synthesized products were separated by SDS-PAGE at neutral pH.

115 The occurrence of IRD is indicated by the accumulation of peptidyl-tRNAs, which are

116 sensitive to RNase A (RN, pre-electrophoresis treatment) or Pth (peptidyl-tRNA

117 hydrolase, added to the translation mixture), but not puromycin (added to the reaction

118 mixture). A typical result with MgtL is presented in Figure 1C, which shows the evident

119 accumulation of a Pth-cleavable peptidyl-tRNA (lanes 1-3, arrows on left). As expected,

120 the no DE/P variant of MgtL did not appreciably generate this product (lanes 4-6). The

121 constructs bearing the other Nt10 sequences also accumulated Pth-cleavable peptidyl-

122 tRNAs in DE/P-dependent manners (Figure 1D). Thus, the $m g t L$-like N-terminal DE/P-

123 enriched sequences in the $E$. coli genes induce IRD in the reconstituted translation system.

N-terminally adjacent nascent polypeptide segments in the exit tunnel counteract IRD in a length-dependent manner.

The translation abortion by the N-terminal DE/P-enriched sequences (Figure 1)

128 led us to investigate whether IRD occurs similarly when a DE/P sequence resides elsewhere, such as internal positions, in a polypeptide chain. To study IRD induction by internal DE/P motifs, we surveyed the E. coli genome and found 2,110 genes, out of the annotated 4,187 ORFs, that contain one or more DE/P-rich segment(s) (having four residues that are either $\mathrm{D}, \mathrm{E}$, or $\mathrm{P}$ in a six amino acid window). In most cases, the motifs were present at internal locations. We engineered the MgtL sequences with the EPDP sequence at various positions relative to the $\mathrm{N}$-terminus, by inserting GFP-derived sequences between the initiator methionine and the EPDP motif. As shown in Figure 2A, the insertion of 238 amino acid residues counteracted the IRD-like translation anomalies at the repositioned EPDP motifs (Figure 2A).

When translating the wild-type $m g t L$, the ribosome accommodates at most a 6 amino acid-long nascent product in its exit tunnel before IRD occurs, leaving the tunnel almost unoccupied. By contrast, the exit tunnel of the ribosome translating the GFPinserted variant of MgtL is fully occupied by the GFP part of the nascent chain when it is translating the repositioned EPDP motif. Thus, we hypothesized that the tunnel-spanning nascent polypeptide preceding the IRD sequence stabilizes the translating ribosome, and 
144 thus counteracts IRD (Figure 2B). We tested this hypothesis by inserting various lengths

145 of sequences from a variety of origins before the EPDP motif of MgtL, as shown in Figure

146 2C. We chose various lengths of the N-terminal regions of GFP, E. coli DnaK and DHFR

147 and evaluated their effects on the EPDP-mediated IRD in living cells. All of the nascent

148 polypeptide segments preceding EPDP lowered the IRD efficiency in length-dependent

149 manners (black line in Figure 2D). It is noteworthy that the IRD-counteracting effects of

150 the inserted segments reached plateaus at a length of $\sim 30$ amino acids. Interestingly, this

15130 amino acid length coincides with the length of the nascent polypeptide that spans the

152 exit tunnel in an extended form (Jenni and Ban, 2003; Voss et al., 2006). When the same

153 proteins were expressed in the bL31 deletion strain, the IRD effect was more pronounced

154 than that in the wild-type host strain, and the protecting effects of the N-terminal

155 polypeptide insertion became somewhat weaker. These results suggest that the possession

156 of an upstream polypeptide sequence exerts an IRD-counteracting effect by stabilizing

157 the translating ribosome. Consistent with this interpretation, we were able to reproduce

158 the polypeptide insertion-dependent IRD alleviation by in vitro assays using the PURE

159 system (Figure 2E), indicating that the IRD-counteraction does not require any factors

160 other than the essential translation factors in this reconstituted system.

161 From these results, we propose that the nascent polypeptide chain within the

162 ribosomal interior space generally contributes to the stability of the translating ribosome

163 and the continuity of translation. To examine if any sequence specificity exists in the

164 ribosome stabilizing ability of the tunnel-occupying polypeptide, we randomly chose 40

165 E. coli genes, and inserted 5 aa and 30 aa parts of their N-terminal regions into the $m g t L$ -

166 lac $Z$ reporter, as described above. We successfully assessed the impacts of the sequences

167 derived from 37 and 21 proteins by an in vivo reporter assay and in vitro translation,

168 respectively (Figure 2F). As shown in the boxplots representation, the 30 aa insertions

169 prevented IRD more effectively than the 5 aa insertions, both in vivo and in vitro. Thus,

170 the polypeptide segments preceding the IRD sequence counteract the IRD in a largely

171 sequence-independent manner, provided that they are long enough ( $\sim 30$ amino acids or

172 longer) to span the exit tunnel.

173

174 Bulky amino acids near the PTC also counteract IRD.

175 We noticed that the 5 aa insertions had a rather broad spectrum of effects on 176 IRD (Figure 2F). The individual in vivo data shown in Figure $2 \mathrm{G}$ indicate that the effects 177 of the 5 aa insertions varied from nearly no effect to almost complete suppression of IRD 178 (Figure 2G). A similar tendency was also observed in the PURE system analysis in vitro 179 (Figure S2A). Thus, not only the nascent chain length but also some property of the amino 
180 acids in the immediate N-terminal vicinity of an IRD sequence can alleviate IRD,

181 probably by stabilizing the $70 \mathrm{~S}$ ribosome. We surveyed various properties of the inserted 182 amino acids and found a positive correlation between the sum of the van der Waals radii 183 of the five inserted amino acids and the IRD-counteracting effects in vivo and in vitro 184 (Figure 2H). Residues with bulkier side chains prevented IRD more effectively than those 185 with smaller ones (Figure 2I). By contrast, the average hydrophobicity showed a poor correlation with the effects (Figure S2B).

We tested the effects of bulky amino acids in the D-rich FLAG tag sequence (DYKDDDK), which exhibited significant IRD (Figure S2C). We mutated its YK part to pairs of identical amino acids, and found that the IRD propensities correlated with the bulkiness of the dipeptides at this position (Figure S2C and S2D). Taken together, bulky amino acid residues just preceding an IRD sequence counteract IRD. Our results suggest that the occupation of the exit tunnel by a nascent chain stabilizes the translating ribosome, by either the "length principle", in which polypeptide chains with diverse sequences of $\sim 30$ residues can work, or the "size principle", in which a short stretch of bulky residues can work.

Multifaceted nascent chain-exit tunnel interactions contribute to the continuous translation elongation.

Previous studies on ribosome arresting peptides revealed that nascent chains interact with the tunnel wall, particularly in the central constriction partially formed by the protein components uL4 and uL22 (Ito and Chiba, 2013) (Figure 3A). We addressed whether the mutations of these proteins affect the two modes of IRD antagonization studied above. We first focused on the role played by the uL22 loop (Figure 3B), and constructed mutant ribosomes with the mutant form lacking the $\beta$-hairpin loop (uL22 2 loop; Figure 3B). We then developed a modified PURE system containing the mutant form of the ribosomes. The results revealed that the effects of the uL22 $\Delta$ loop mutation differ, depending on the preceding amino acid lengths. Whereas the uL22 mutated ribosome was almost indistinguishable from the wild-type ribosome in the IRD profile of the EPDP constructs with the upstream 5 amino acids (Figure 3C, Figure S3, orange line), the absence of the loop enhanced IRD when the upstream region contained 30 amino acids (Figure 3C, Figure S3).

This substrate-dependent effect of the uL22 mutation is in sharp contrast to our

214 IRD (lowered the translation continuity), with both templates having the 5 aa and 30 aa 215 insertions (Figure 3C. For individual data, see also Figure S3, compare black and blue 
216 lines). These results indicate that the $\beta$-hairpin loop of uL22 in the wild-type ribosome

217 plays a role in counteracting IRD, but only in the presence of the tunnel-spanning

218 upstream polypeptide region (Figure 3B). By contrast, bulky side chains near the PTC 219 can stabilize the ribosome irrespective of the tunnel status (Figure 2I).

220 We further explored the relationships between the exit tunnel occupation and the

221 stability of the translating ribosome, using various lengths of the N-terminal regions of

222 DnaK, GrpE and NuoN that were attached N-terminally to the EPDP motif. The wild type

223 and uL22 $\Delta$ loop ribosomes translated the EPDP constructs with the 5 amino acid- and the

22410 amino acid-upstream regions with almost the same degrees of IRD, which differed

225 depending on the sequences (Figure 3D, compare black and orange lines). However, the

226 uL22 $\Delta$ loop ribosomes showed an increased IRD when 20 or more amino acids were

227 present before the EPDP (Figure 3D, orange lines). Since previous studies suggested that

228 the uL22 $\beta$-hairpin loop interacts with the nascent polypeptides at a 10-20 aa distance

229 from the PTC (Bhushan et al., 2011; Seidelt et al., 2009; Shanmuganathan et al., 2019;

230 Sohmen et al., 2015; Su et al., 2017), our data suggest that the interactions between the

231 nascent polypeptide and the tunnel constriction site contribute to the elongation continuity

232 beyond the IRD sequence, perhaps by changing the stability of the ribosome.

233 We next studied the effect of the other ribosomal tunnel mutation on IRD. We

234 used uL23 $\Delta$ loop with the deletion of the uL23 signaling loop, which otherwise extends

235 into the PTC-distal part of the exit tunnel (Bornemann et al., 2008, Figure 3E). We

236 translated RpoD, YihI and the artificial GFP-10E (Chadani et al., 2017), which have

237 acidic stretches starting from their $191^{\text {st }}, 147^{\text {th }}$ and $379^{\text {th }}$ residues, respectively, using the

238 PURE system with the mutated ribosomes (Figure 3F). The new substrate proteins with

239 their acidic regions farther away from the N-termini exhibited rather weak IRD when

240 translated with the wild-type ribosome, while the IRD was enhanced by the absence of

241 bL31 (Figure 3F). As we observed with the uL22 $\Delta$ loop mutant, the deletion of the uL23

242 signaling loop enhanced IRD in all cases, suggesting that the uL23 loop in the wild-type

243 ribosome contributes to the ribosome stability (Figure 3F). By contrast, the N-terminal

244 IRD, without an insertion, was not affected by the tunnel mutations of the ribosome

245 (Figure 3G). The latter results are expected, because these constructs are subject to

246 translation discontinuation before accessing the uL22 and the uL23 tips in the tunnel

247 (Figure 3B and 3E). These results suggest that nascent chains spanning the tunnel interact

248 with the tunnel-exposed protein components of the ribosome to stabilize the translating

249 ribosome and ensure translation continuity, irrespective of the amino acids being

250 polymerized at the PTC. The involvement of multiple ribosomal proteins suggests that 
multiple interactions, which alone may be subtle, cumulatively contribute to the continuous translation.

\section{Proteome-wide amino acid distribution bias that minimizes the IRD risk.}

The features of the non-uniform translation revealed above might have affected the amino acid composition of proteins during the evolution of ORFs. Specifically, the $\mathrm{N}$-terminal regions of ORFs might have avoided the enrichment of $\mathrm{D}, \mathrm{E}$, and $\mathrm{P}$ and preferred amino acid residues with bulky side chains to allow for robustly continuous translation elongation. We addressed this question by bioinformatics. The appearance of more than three D, E or P residues in ten-residue moving windows is lower for the Nterminal 20-30 positions of the E. coli ORFs than the following positions (Figure 4A). A previous bioinformatics analysis also showed a similar decline of negative charges at the

263 beginning of the ORFs (Dao Duc and Song, 2018). We also observed that the van der

264 Waals radii of amino acids in the N-terminal regions are larger than those in the other 265 parts of ORFs (Figure 4B). Such biased amino acid composition features of the N266 terminal regions are widely conserved among bacterial species, including Bacillus subtilis, 267 Staphylococcus aureus and Thermus thermophilus (Figure S4). We suggest that the amino acid distribution biases were acquired during evolution as IRD-counteracting measures.

\section{Proteome-wide IRD and its counteraction revealed by ribosome profiling}

In spite of the statistical tendency revealed above, the N-terminal regions of ORFs still have many DE/P- or small amino acid-rich regions (Figure 4), with some of the former inducing IRD (Figure 1). To capture IRD globally, we performed ribosome profiling (Ribo-seq, Ingolia et al., 2009) and monitored the ribosomal occupancy along mRNAs. We expected that the translation of ORFs with a DE/P-enriched sequence in the $\mathrm{N}$-terminal regions would cause a decline in the ribosome occupancy (the number of ribosome-protected RNA fragments, RPFs) in the following 3' region, by the stochastic release of the ribosomes from the mRNA due to IRD (Figure 5A). To survey ORFs with the above-mentioned characteristic patterns of ribosome distribution, we compared the sum of the RPF counts at the first 10 codons with those at the following 10 codons (Figure 5B). We first focused on $y b e X$ as one of the ORFs that exhibited the above mentioned Ribo-seq patterns. The N-terminal segment (MSDDNSHSSDT) of $y b e X$ is enriched in Asp and small-sized amino acids (Ser and Thr). The RPFs from the $y b e X$ mRNA decreased after the $11^{\text {th }}$ codon (Figure 5C). A biochemical analysis showed that a 
286 peptidyl-tRNA accumulated in an Asp-dependent manner during the in vitro translation

287 of $y b e X$ (Figure S5A), substantiating the occurrence of an elongation cessation.

288 We next analyzed the global Ribo-seq data to compare the sum of the RPF

289 counts at the first 10 codons with those at the following 10 codons. We extracted the

290 ORFs enriched in DE/P residues in their N-terminal regions, and then classified them

291 further into those enriched in small amino acids and those enriched in bulky amino acids

292 (Figure S5B). The analysis revealed that $\sim 16 \%$ of RPFs were lost after 10 codons (30

293 nucleotides) for ORFs with an abundance of small-sized amino acids in their N-terminal

294 regions (Figure 5D, panel 1). The bL31-deleted cells showed more pronounced decreases

295 in RPFs after the $10^{\text {th }}$ codon (Figure 5D, panel 2), further supporting our contention that

296 the global low-occupancy of the ribosomes after the risky sequences represents IRD. By

297 contrast, we did not observe any decrease in the RPF for ORFs containing DE/P-rich and

298 large-sized amino acids in the N-terminal region (Figure 5D), consistent with our findings

299 that bulky amino acids antagonize IRD.

300 We next undertook a more systematic survey of the N-terminal regions of $E$. coli

301 proteins for the occurrence of IRD-like phenomena. In this analysis, we searched for

302 sequences enriched in DE/P and small-sized residues in the successive 10 amino acid

303 windows moving from the $\mathrm{N}$ - to $\mathrm{C}$-terminal regions, as schematically shown in Figure 5E

304 (a)-(e). We then analyzed the numbers of RPFs across the putative IRD sequences. We

305 found $15-20 \%$ declines when the windows starting at the second or the $12^{\text {th }}$ codons were

306 examined (Figure 5E, panel 1 (a), (b)). Such tendencies were gradually lost for windows

307 moving further toward the 3 ' direction, in which the elongating nascent polypeptide

308 should have possessed successively longer sequences preceding the IRD determinant.

309 The results with the windows of 22-31 (c), 32-41 (d) and at the C-terminus (e) indicated

310 that the ribosomes essentially continue to translate these regions even when they

311 encounter DE/P- and smaller residue-enriched segments. The bL31 deletion uniformly

312 enhanced the decline of RPFs, again supporting that the elongation discontinuations were

313 due to IRD (Figure 5E, panel 2). We did not observe equivalent decreases in RPFs when

314 we extracted the DE/P-enriched sequences that were associated with bulky amino acid

315 residues (Figure S5C), suggesting that the nascent chain "length"- and amino acids

316 "bulkiness"-dependent mechanisms synergistically affect the translation continuity.

317 Collectively, our Ribo-seq analysis revealed that DE/P-enriched segments that also

318 contained small-sized residues generally induce IRD when they are present at N-terminal

319 regions. 
321 Tunnel-occupying nascent polypeptides stabilize the translating $70 \mathrm{~S}$ ribosome by 322 bridging the $50 \mathrm{~S}$ and the $30 \mathrm{~S}$ subunits.

323 Our findings that the nascent polypeptide spanning the exit tunnel counteracts 324 the IRD-mediated translation discontinuation strongly suggested that the tunnel325 occupying nascent polypeptide functions as an inter-subunit bridge, like the bL31326 supported B1b peripheral bridge. We performed a sucrose gradient centrifugation 327 analysis to assess the stability of the $70 \mathrm{~S}$ ribosome, with or without the nascent 328 polypeptide within the tunnel. As reported previously (Chadani et al., 2017; Fischer et al., 329 2015; Lilleorg et al., 2017; Ueta et al., 2017), the deletion of bL31 destabilizes the 70S 330 complex structure, resulting in partial dissociation into the $30 \mathrm{~S}$ and $50 \mathrm{~S}$ subunits in cell 331 lysates (Figure 6A). We found that the small amount of the $70 \mathrm{~S}$ complex remaining in 332 the $\Delta$ bL31 cell lysate was completely eliminated by a treatment with puromycin. This 333 drug cleaves peptidyl-tRNA to produce peptidyl-puromycin, which neither interacts with 334 mRNA nor remains ribosome-associated (blue line). By contrast, chloramphenicol, which 335 blocks translation elongation, preserved the 70S complex (red line). These results are 336 consistent with the notion that the nascent polypeptide stabilizes the 70S structure.

337 To substantiate the concept that the nascent polypeptide stabilizes the association 338 of the large and small ribosomal subunits more directly, we compared the stabilities of 339 the 70S ribosomes with a tunnel-traversing nascent polypeptidyl-tRNA and those that had 340 lost the intact polypeptidyl-tRNA. We isolated the 70S ribosomes in the process of 341 translation by treating a polysome preparation with micrococcal nuclease, which cleaves 342 unoccupied mRNA. The resulting nascent chain-ribosome complexes exhibited the $\sim 70 \mathrm{~S}$ 343 sedimentation speed. We then treated the isolated $70 \mathrm{~S}$ complex with puromycin and 344 centrifuged the samples again (Figure $6 \mathrm{~B}$ ). Wild type ribosomes sedimented at $\sim 70 \mathrm{~S}$ 345 irrespective of the puromycin treatment (Figure 6C, panels 1 and 3). By contrast, the 346 bL31-lacking ribosomes were dissociated by the puromycin treatment into the 30S and 347 50S subunits (Figure 6C, panel 4). Thus, the nascent polypeptides (peptidyl-tRNAs) 348 within the exit tunnel stabilize the translating $70 \mathrm{~S}$ ribosome complex even in the absence 349 of the bL31 bridge. It should be noted that the nascent polypeptides within the $70 \mathrm{~S}$ 350 ribosomes in this experiment consisted of a mixture of endogenous gene products, which 351 do not necessarily contain IRD sequences around the PTC. 


\section{Discussion}

Recent advances in translation studies have revealed the widespread occurrence of noncanonical translation progression (Dever et al., 2020; Samatova et al., 2021). We showed that elongation pausing is frequent in E. coli, by capturing intermediate molecules with tRNAs at their ends (Chadani et al., 2016). More recently, we discovered the IRD phenomena (Chadani et al., 2017), in which the translation of certain acidic sequences, sometimes containing a proline, leads to the destabilization of the $70 \mathrm{~S}$ ribosome and translation cessation without involving a stop codon. In the present study, we addressed the generality, the position effects, and the sequence context-dependence of potentially IRD-inducing DE/P-rich motifs in E. coli, featuring both model constructs and the native genes in the genome. Our results showed that nascent polypeptides in the interior of the translating ribosome have regulatory roles over the stability of the engaged ribosomes. In particular, we found that the nascent chain exerts stabilizing effects on the 70S structure, thus compensating for the potential IRD induction by a risky DE/P-rich part in the following region of the same polypeptide.

In the translocation step of translation, the large and small subunits undergo a relative rotation, which their association must withstand to produce a protein product (Bock et al., 2015; Horan and Noller, 2007; Voorhees and Ramakrishnan, 2013). Thus, the ribosome is equipped with multiple bridges, including bL31, that tether the large and small subunits in the functional monosome (Chadani et al., 2017; Fischer et al., 2015; Lilleorg et al., 2017; Ueta et al., 2017). We have shown that the growing nascent chain itself also contributes to the stabilization of the protein synthesizing machine. Although we initially found the stabilization effect of nascent chains from their counteraction to IRD, our sedimentation analysis using cell lysates (Figure 6) suggested that endogenous sources of nascent polypeptidyl-tRNA, not necessarily combined with a DE/P sequence, stabilize the 70S structure. Thus, we propose that the ribosome stabilization by the tunneloccupying nascent chain represents a general life principle.

Earlier studies reported the peptidyl-tRNA-dependent stabilization of the 70S structure under $\mathrm{Mg}^{2+}$-depleted conditions (Duin et al., 1970; Kohler et al., 1968; Ron et al., 1968; Schlessinger et al., 1967). Other reports described the destabilization of a nascent chain-free ribosome complex under high ionic conditions (Blobel and Sabatini, 1971; Zylber and Penman, 1970). Whereas these observations complement our findings, they were made before the discovery of the ribosomal exit tunnel. In addition, cryptic and apparently risky sequences like the IRD-motifs were unknown, and thus the physiological significance of the early findings remained unexplored. Our work can therefore be 
390 dynamics. Our results have engendered the emergence of the novel concept that the 391 nascent products of translation, in conjunction with the exit tunnel, contribute to the stability of the ribosome in action. We named it $\underline{T}$ unnel-occupying nascent $\underline{P}$ olypeptidedependent $\underline{S}$ tabilization (TPS). TPS enables the ribosome to be a universal translation machine that can synthesize polypeptides of any sequence without discontinuation to produce the life-supporting proteome of the species. The C-terminal end of a nascent polypeptide is covalently tethered to a tRNA that interacts with the $30 \mathrm{~S}$ component and the mRNA in the $30 \mathrm{~S}$ decoding center. Meanwhile, its more N-terminal region resides in the 50S subunit, entering into and occupying the exit tunnel. Thus, a nascent polypeptide bridges the small and large subunits. TPS represents a self-stabilization mechanism to prevent the dissociation of the translating ribosome.

Despite the potentially deleterious outcomes, dozens of $E$. coli genes with N402 terminal DE/P-rich sequences still evoke IRD. Why do these genes keep IRD sequences, which destabilize the ribosome? One possibility is that cells harness IRD as a regulatory scheme at the translational and/or transcriptional levels. A typical example might be found in the translation of MgtL, which serves as a $\mathrm{Mg}^{2+}$ sensor for regulation of the MgtA $\mathrm{Mg}^{2+}$ transporter (Chadani et al., 2017). Our present ribosome profiling and biochemical experiments revealed that the expression of $y b e X$ ( $\operatorname{cor} C$, Giménez-Mascarell et al., 2019), a putative $\mathrm{Mg}^{2+} / \mathrm{Co}^{2+}$ efflux pump, can be repressed in an IRD-dependent manner. An increased $\mathrm{Mg}^{2+}$ concentration in the cell would stabilize the ribosome and decrease the IRD-mediated premature translation cessation, leading to the enhancement of the $y b e X$ expression and the consequent $\mathrm{Mg}^{2+}$ efflux. It is also conceivable that the $\mathrm{N}-$ terminal IRD sequences are advantageous for cells to respond to certain stresses other 413 than changes in the $\mathrm{Mg}^{2+}$ concentration that affect the ribosome stability (Rasouly and 414 Ron, 2009). The N-terminal IRD sequences might be a hidden code for the "ribosome stability regulon" that reshapes the proteome upon emergency conditions.

Nascent polypeptides preceding the DE/P-rich sequences antagonize IRD. In the

417 "length"-dependent but sequence-independent mode of the stabilization, the nascent chain 418 might act as an intersubunit scaffold. The deletion of either the uL22 $\beta$-hairpin loop or 419 the uL23 signaling loop weakens the effect but does not completely abolish it, suggesting 420 that multiple weak interactions between the interior of the tunnel and the nascent 421 polypeptide contribute to the stabilization. The diverse effects of the ribosomal mutations affecting different ribosomal proteins, such as uL22 and uL23, imply that the ribosome and the nascent chain communicate with each other by the local diversities, producing cumulative weak interactions to perform the biological role of the "peptidyl-tRNA 
425 bridge", although such interactions poorly inhibit the nascent chain movement through 426 the tunnel.

These non-uniform features of the interactions remind us of the interactions between the tunnel and ribosome arresting peptides (RAPs, Ito and Chiba, 2013). Each part of the tunnel architecture differently interacts with each of the RAP sequences to arrest translation. For example, the alternation of either of uL22 Gly91, Ala93 or the 23S rRNA A2058 residue attenuates the translational arrest induced by RAP in E. coli SecM, but not that of E. coli TnaC (Cruz-Vera et al., 2005; Nakatogawa and Ito, 2002). In this respect, RAPs are similar to the peptides involved in TPS, but differ in that RAPs require specific polypeptide sequences at a particular site within the tunnel. However, too many specific interactions between the tunnel and the nascent polypeptide would inhibit its passage through the tunnel, resulting in translational arrest events. From this viewpoint, TPS and translation arrest share the same principle, but with different strengths. TPS promises the continuity of translation, but too much stabilization might be one of the driving forces of translational arrest. The widespread occurrence of translational pausing during the synthesis of the E. coli proteome might reflect such interactions within the tunnel (Chadani et al., 2016). Since the translational arrest events have physiological impacts, modulation of TPS could influence the elongation velocity, leading to cotranslational control of gene expression.

The bulkiness, defined as the van der Waals radii, of the amino acids in the nascent chain adjacent to PTC also counteracted IRD. It is likely that the "bulkiness"dependent stabilization plays a more important role during the early stage of translation, at which the "length" works poorly. The "length"-dependent mechanism apparently lacks a preference for amino acid sequences, but its effect would be insufficient until the nascent polypeptide fulfills the tunnel (no less than 20 aa). In contrast, the "bulkiness"dependent stabilization necessitating larger residues restricts the usage of amino acids, unlike the "length"-dependent one. We assume that these two stabilization mechanisms complementarily compensate for their respective insufficiencies to avoid nonproductive premature termination. The strong biases on disfavored DE/P-rich sequences and favored bulky amino acids in the N-terminal regions of bacterial ORFs are in agreement with this assumption (Figure 4 and Figure S4). Many previous studies have pointed out that the sequence features just adjacent to the start codon significantly influence the efficiency of protein biosynthesis (Allert et al., 2010; Goodman et al., 2013; Kudla et al., 2009;

458 Osterman et al., 2020; Salis et al., 2009; Sprengart et al., 1996; Verma et al., 2019). 459 Considering the "bulkiness" of the nascent polypeptide when interpreting the datasets 460 might disclose new landscapes from previous studies. 
The "size (bulkiness)"-dependence indicates the relationship between the geometry of the tunnel structure and the stabilization mechanism. Lu and colleagues reported the "size"-dependent rearrangement of the nascent polypeptide at the eukaryotic uL4-uL22 constriction site, indicating that larger residues have more interaction chances than smaller ones (Lu et al., 2011). The translation of consecutive tryptophan residues, the largest natural amino acid, inhibits elongation by the eukaryotic ribosome, in agreement with this observation (Dimitrova et al., 2009). The prokaryotic uL4-uL22 constriction site is slightly broader than that of eukaryotes (Dao Duc et al., 2019), and tryptophan repeats do not induce translational anomalies in E. coli (Chadani et al., 2017). However, the prokaryotic tunnel forms another constriction site at the entrance of the tunnel, which is as narrow as the eukaryotic uL4-uL22 constriction site (tunnel radii are below $4 \AA$, Dao Duc et al., 2019; Fischer et al., 2015). Taken together with these facts, we assume that the squeezing of bulky residues by this "entry constriction site" represses the unwinding of the growing products around the PTC, thus stabilizing the 70S complex. Other bacterial ribosomes show similar architectural characteristics, supporting this assumption.

Finally, this study provides an answer to the naive question of why the ribosome has an exit tunnel for the nascent polypeptide. A tunnel may not be needed, if the essential function of the ribosome is simply to polymerize the polypeptides. Stabilization of the translating ribosome by preparing the tunnel for occupation with its own product would be a positive feedback system to continue the elongation. There might be a trade-off between the stabilization effects and the obstacles of translation elongation. The tunnel architecture, which is less than 30 40 aa in length, might represent a compromise for these characteristics.

The schemes of the two stabilizing mechanisms provide a glimpse of the molecular details of IRD. It is likely that "length"- and "size (bulkiness)"-dependent stabilization commonly minimizes the fluctuations of polypeptidyl-tRNAs within the ribosome complex. Taken together with the putative negative-negative repulsion between the D/E residues and the rRNA component of the tunnel wall (Nissen et al., 2000), we assume that IRD interrupts the correct positioning of the polypeptidyl-tRNA, resulting in a disruption of the codon-anticodon interactions and the following subunit dissociation. Understanding such aspects of the ribosomes will clarify the basis of ribosomal functions and the secondary genetic codes hidden within amino acid sequences. 


\section{Author Contributions}

496 YC and NS performed experiments; YC, NS, TN, YI, SI and HT conceived the study, 497 designed experiments and analyzed the results; YC and HT supervised the entire project;

498 YC and HT wrote the manuscript.

499

\section{Acknowledgments}

501 We thank Koreaki Ito for critical reading and editing of the manuscript, Eri Uemura for 502 technical support, the Bio-support Center at Tokyo Tech for DNA sequencing, and The 503 National BioResource Project, E. coli (National Institute of Genetics, Japan) for 504 providing the ASKA library clones and Keio collection strains. This work was supported 505 by MEXT Grants-in-Aid for Scientific Research (Grant Numbers JP26116002, 506 JP18H03984, JP20H05925 to HT, JP17H05679, JP17H04998, JP19K22406, 507 JP20H05784 to SI, JP17K15062, JP19K16038 to YC), and a grant from the Ohsumi 508 Frontier Science Foundation to YC.

509 


\section{References}

Allert, M., Cox, J.C., and Hellinga, H.W. (2010). Multifactorial Determinants of Protein Expression in Prokaryotic Open Reading Frames. J Mol Biol 402, 905-918.

Bhushan, S., Hoffmann, T., Seidelt, B., Frauenfeld, J., Mielke, T., Berninghausen, O., Wilson, D.N., and Beckmann, R. (2011). SecM-Stalled Ribosomes Adopt an Altered Geometry at the Peptidyl Transferase Center. PLoS Biology 9, e1000581.

Blobel, G., and Sabatini, D. (1971). Dissociation of Mammalian Polyribosomes into Subunits by Puromycin. Proc National Acad Sci 68, 390-394.

Bock, L.V., Blau, C., Vaiana, A.C., and Grubmüller, H. (2015). Dynamic contact network between ribosomal subunits enables rapid large-scale rotation during spontaneous translocation. Nucleic Acids Res 43, 6747-6760.

Bornemann, T., Jöckel, J., Rodnina, M.V., and Wintermeyer, W. (2008). Signal sequence-independent membrane targeting of ribosomes containing short nascent peptides within the exit tunnel. Nat Struct Mol Biol 15, 494-499.

Buskirk, A.R., and Green, R. (2017). Ribosome pausing, arrest and rescue in bacteria and eukaryotes. Philosophical Transactions Royal Soc B Biological Sci 372, 20160183.

Chadani, Y., Niwa, T., Chiba, S., Taguchi, H., and Ito, K. (2016). Integrated in vivo and in vitro nascent chain profiling reveals widespread translational pausing. Proc Natl Acad Sci USA 113, E829-38.

Chadani, Y., Niwa, T., Izumi, T., Sugata, N., Nagao, A., Suzuki, T., Chiba, S., Ito, K., and Taguchi, H. (2017). Intrinsic Ribosome Destabilization Underlies Translation and Provides an Organism with a Strategy of Environmental Sensing. Mol Cell 68, 528539.e5.

Chiba, S., Lamsa, A., and Pogliano, K. (2009). A ribosome-nascent chain sensor of membrane protein biogenesis in Bacillus subtilis. EMBO J 28, 3461-3475.

Choi, J., Grosely, R., Prabhakar, A., Lapointe, C.P., Wang, J., and Puglisi, J.D. (2015). How Messenger RNA and Nascent Chain Sequences Regulate Translation Elongation. Annu Rev Biochem 87, 421-449.

Cruz-Vera, L.R., Rajagopal, S., Squires, C., and Yanofsky, C. (2005). Features of Ribosome-Peptidyl-tRNA Interactions Essential for Tryptophan Induction of tna Operon Expression. Mol Cell 19, 333-343.

Dao Duc, K., and Song, Y.S. (2018). The impact of ribosomal interference, codon usage, and exit tunnel interactions on translation elongation rate variation. PLOS Genet 14 , e1007166. 
Dao Duc, K., Batra, S.S., Bhattacharya, N., D. Cate, J.H., and Song, Y.S. (2019). Differences in the path to exit the ribosome across the three domains of life. Nucleic Acids Res 47, 4198-4210.

Das, G., and Varshney, U. (2006). Peptidyl-tRNA hydrolase and its critical role in protein biosynthesis. Microbiology 152, 2191-2195.

Datsenko, K.A., and Wanner, B.L. (2000). One-step inactivation of chromosomal genes in Escherichia coli K-12 using PCR products. Proc Natl Acad Sci USA 97, 6640-6645.

Dever, T.E., Ivanov, I.P., and Sachs, M.S. (2020). Conserved Upstream Open Reading Frame Nascent Peptides that Control Translation. Annu Rev Genet 54, 1-28.

Dimitrova, L.N., Kuroha, K., Tatematsu, T., and Inada, T. (2009). Nascent Peptidedependent Translation Arrest Leads to Not4p-mediated Protein Degradation by the Proteasome. J Biol Chem 284, 10343-10352.

Duin, J. van, Dieijen, G.V., Dieijen, G., Knippenberg, P.H. van, and Bosch, L. (1970). Different Species of 70S Ribosomes of Escherichia coli and their Dissociation into Subunits. Eur J Biochem 17, 433-440.

Fischer, N., Neumann, P., Konevega, A.L., Bock, L.V., Ficner, R., Rodnina, M.V., and Stark, H. (2015). Structure of the E. coli ribosome-EF-Tu complex at $<3 \AA$ resolution by Cs-corrected cryo-EM. Nature 520, 567-570.

Gall, A.R., Datsenko, K.A., Figueroa-Bossi, N., Bossi, L., Masuda, I., Hou, Y.-M., and Csonka, L.N. (2016). $\mathrm{Mg}^{2+}$ regulates transcription of mgtA in Salmonella Typhimurium via translation of proline codons during synthesis of the MgtL peptide. Proc National Acad Sci 113, 15096-15101.

Giménez-Mascarell, P., González-Recio, I., Fernández-Rodríguez, C., Oyenarte, I., Müller, D., Martínez-Chantar, M.L., and Martínez-Cruz, L.A. (2019). Current Structural Knowledge on the CNNM Family of Magnesium Transport Mediators. Int J Mol Sci 20, 1135 .

Gong, F., and Yanofsky, C. (2002). Instruction of Translating Ribosome by Nascent Peptide. Science 297, 1864-1867.

Goodman, D.B., Church, G.M., and Kosuri, S. (2013). Causes and Effects of NTerminal Codon Bias in Bacterial Genes. Science 342, 475-479.

Guzman, L.M., Belin, D., Carson, M.J., and Beckwith, J. (1995). Tight regulation, modulation, and high-level expression by vectors containing the arabinose PBAD promoter. J Bacteriol 177, 4121-4130. 
Han, P., Shichino, Y., Schneider-Poetsch, T., Mito, M., Hashimoto, S., Udagawa, T., Kohno, K., Yoshida, M., Mishima, Y., Inada, T., and Iwasaki, S. (2020). Genome-wide Survey of Ribosome Collision. Cell Reports 31, 107610.

Horan, L.H., and Noller, H.F. (2007). Intersubunit movement is required for ribosomal translocation. Proc Natl Acad Sci USA 104, 4881-4885.

Ieong, K.-W., Uzun, Ü., Selmer, M., and Ehrenberg, M. (2016). Two proofreading steps amplify the accuracy of genetic code translation. Proc Natl Acad Sci USA 113, 1374413749.

Inada, T. (2017). The Ribosome as a Platform for mRNA and Nascent Polypeptide Quality Control. Trends Biochem Sci 42, 5-15.

Ingolia, N.T., Ghaemmaghami, S., Newman, J.R.S., and Weissman, J.S. (2009). Genome-wide analysis in vivo of translation with nucleotide resolution using ribosome profiling. Science 324, 218-223.

Ishii, E., Chiba, S., Hashimoto, N., Kojima, S., Homma, M., Ito, K., Akiyama, Y., and Mori, H. (2015). Nascent chain-monitored remodeling of the Sec machinery for salinity adaptation of marine bacteria. Proc Natl Acad Sci USA 112, E5513-E5522.

Ito, K., and Chiba, S. (2013). Arrest Peptides: Cis-Acting Modulators of Translation. Annu Rev Biochem 82, 171-202.

Jenni, S., and Ban, N. (2003). The chemistry of protein synthesis and voyage through the ribosomal tunnel. Curr Opin Struc Biol 13, 212-219.

Keiler, K.C. (2008). Biology of trans-Translation. Microbiology 62, 133-151.

Kitagawa, M., Ara, T., Arifuzzaman, M., Ioka-Nakamichi, T., Inamoto, E., Toyonaga, H., and Mori, H. (2006). Complete set of ORF clones of Escherichia coli ASKA library (A Complete Set of E. coli K-12 ORF Archive): Unique Resources for Biological Research. DNA Res 12, 291-299.

Kohler, R.E., Ron, E.Z., and Davis, B.D. (1968). Significance of the free 70 s ribosomes in Escherichia coli extracts. J Mol Biol 36, 71-82.

Kudla, G., Murray, A.W., Tollervey, D., and Plotkin, J.B. (2009). Coding-Sequence Determinants of Gene Expression in Escherichia coli. Science 324, 255-258.

Lilleorg, S., Reier, K., Remme, J., and Liiv, A. (2017). The Intersubunit Bridge B1b of the Bacterial Ribosome Facilitates Initiation of Protein Synthesis and Maintenance of Translational Fidelity. J Mol Biol 429, 1067-1080. 
Lu, J., Hua, Z., Kobertz, W.R., and Deutsch, C. (2011). Nascent Peptide Side Chains Induce Rearrangements in Distinct Locations of the Ribosomal Tunnel. J Mol Biol 411, 499-510.

McGlincy, N.J., and Ingolia, N.T. (2017). Transcriptome-wide measurement of translation by ribosome profiling. Methods 126, 112-129.

Miller, J.H. (1972). Experiments in Molecular Genetics (Cold Spring Harbor Laboratory).

Nakatogawa, H., and Ito, K. (2002). The Ribosomal Exit Tunnel Functions as a Discriminating Gate. Cell 108, 629-636.

Nissen, P., Hansen, J., Ban, N., Moore, P.B., and Steitz, T.A. (2000). The Structural Basis of Ribosome Activity in Peptide Bond Synthesis. Science 289, 920-930.

Noel, J.K., and Whitford, P.C. (2016). How EF-Tu can contribute to efficient proofreading of aa-tRNA by the ribosome. Nat Commun 7, 13314.

Ohashi, H., Shimizu, Y., Ying, B.-W., and Ueda, T. (2007). Efficient protein selection based on ribosome display system with purified components. Biochem Biophys Res Commun 352, 270-276.

Onouchi, H., Nagami, Y., Haraguchi, Y., Nakamoto, M., Nishimura, Y., Sakurai, R., Nagao, N., Kawasaki, D., Kadokura, Y., and Naito, S. (2005). Nascent peptidemediated translation elongation arrest coupled with mRNA degradation in the CGS1 gene of Arabidopsis. Gene Dev 19, 1799-1810.

Osterman, I.A., Chervontseva, Z.S., Evfratov, S.A., Sorokina, A.V., Rodin, V.A., Rubtsova, M.P., Komarova, E.S., Zatsepin, T.S., Kabilov, M.R., Bogdanov, A.A., Gelfand, M.S., Dontsova, O.A., and Sergiev, P.V. (2020). Translation at first sight: the influence of leading codons. Nucleic Acids Res 48, 6931-6942.

Park, S.-Y., Cromie, M.J., Lee, E.-J., and Groisman, E.A. (2010). A Bacterial mRNA Leader that Employs Different Mechanisms to Sense Disparate Intracellular Signals. Cell 142, 737-748.

Rasouly, A., and Ron, E.Z. (2009). Interplay between the heat shock response and translation in Escherichia coli. Res Microbiol 160, 288-296.

Requião, R.D., Souza, H.J.A. de, Rossetto, S., Domitrovic, T., and Palhano, F.L. (2016). Increased ribosome density associated to positively charged residues is evident in ribosome profiling experiments performed in the absence of translation inhibitors. RNA Biol 13, 561-568. 
Rodnina, M.V. (2016). The ribosome in action: Tuning of translational efficiency and protein folding. Protein Sci 25, 1390-1406.

Rodnina, M.V. (2018). Translation in Prokaryotes. Cold Spring Harb Perspect Biol 10, a032664.

Rodnina, M.V., and Wintermeyer, W. (2001). Fidelity of aminoacyl-tRNA selection on the ribosome: kinetic and structural mechanisms. Annu Rev Biochem 70, 415-435.

Rodnina, M.V., and Wintermeyer, W. (2016). Protein Elongation, Co-translational Folding and Targeting. J Mol Biol 428, 2165-2185.

Ron, E.Z., Kohler, R.E., and Davis, B.D. (1968). Magnesium ion dependence of free and polysomal ribosomes from Escherichia coli. J Mol Biol 36, 83-89.

Sabi, R., and Tuller, T. (2015). A comparative genomics study on the effect of individual amino acids on ribosome stalling. BMC Genomics 16, S5.

Salis, H.M., Mirsky, E.A., and Voigt, C.A. (2009). Automated design of synthetic ribosome binding sites to control protein expression. Nat Biotechnol 27, 946-950.

Samatova, E., Daberger, J., Liutkute, M., and Rodnina, M.V. (2021). Translational Control by Ribosome Pausing in Bacteria: How a Non-uniform Pace of Translation Affects Protein Production and Folding. Front Microbiol 11, 619430.

Schlessinger, D., Mangiarotti, G., and Apirion, D. (1967). The formation and stabilization of 30S and 50S ribosome couples in Escherichia coli. Proc Natl Acad Sci USA $58,1782-1789$.

Seidelt, B., Innis, C.A., Wilson, D.N., Gartmann, M., Armache, J.-P., Villa, E., Trabuco, L.G., Becker, T., Mielke, T., Schulten, K., Steitz, T.A., and Beckmann, R. (2009). Structural Insight into Nascent Polypeptide Chain-Mediated Translational Stalling. Science 326, 1412-1415.

Shanmuganathan, V., Schiller, N., Magoulopoulou, A., Cheng, J., Braunger, K., Cymer, F., Berninghausen, O., Beatrix, B., Kohno, K., von Heijne, G., and Beckmann, R. (2019). Structural and mutational analysis of the ribosome-arresting human XBP1u. eLife 8 , e46267.

Sharma, S., Kaushik, S., Sinha, M., Kushwaha, G.S., Singh, A., Sikarwar, J., Chaudhary, A., Gupta, A., Kaur, P., and Singh, T.P. (2014). Structural and functional insights into peptidyl-tRNA hydrolase. Biochimica et Biophysica Acta 1844, 12791288. 
Shimizu, Y., Inoue, A., Tomari, Y., Suzuki, T., Yokogawa, T., Nishikawa, K., and Ueda, T. (2001). Cell-free translation reconstituted with purified components. Nat Biotechnol 19, 751-755.

Sohmen, D., Chiba, S., Shimokawa-Chiba, N., Innis, C.A., Berninghausen, O., Beckmann, R., Ito, K., and Wilson, D.N. (2015). Structure of the Bacillus subtilis 70S ribosome reveals the basis for species-specific stalling. Nat Commun 6, 1-10.

Sprengart, M.L., Fuchs, E., and Porter, A.G. (1996). The downstream box: an efficient and independent translation initiation signal in Escherichia coli. EMBO J 15, 665-674.

Su, T., Cheng, J., Sohmen, D., Hedman, R., Berninghausen, O., von Heijne, G., Wilson, D.N., and Beckmann, R. (2017). The force-sensing peptide VemP employs extreme compaction and secondary structure formation to induce ribosomal stalling. eLife 6 , e25642.

Ueta, M., Wada, C., Bessho, Y., Maeda, M., and Wada, A. (2017). Ribosomal protein L31 in Escherichia coli contributes to ribosome subunit association and translation, whereas short L31 cleaved by protease 7 reduces both activities. Genes Cells 22, $452-$ 471.

Verma, M., Choi, J., Cottrell, K.A., Lavagnino, Z., Thomas, E.N., Pavlovic-Djuranovic, S., Szczesny, P., Piston, D.W., Zaher, H.S., Puglisi, J.D., and Djuranovic, S. (2019). A short translational ramp determines the efficiency of protein synthesis. Nat Commun 10, 5774 .

Voorhees, R.M., and Ramakrishnan, V. (2013). Structural Basis of the Translational Elongation Cycle. Annu Rev Biochem 82, 203-236.

Voss, N.R., Gerstein, M., Steitz, T.A., and Moore, P.B. (2006). The Geometry of the Ribosomal Polypeptide Exit Tunnel. J Mol Biol 360, 893-906.

Wilson, D.N., and Beckmann, R. (2011). The ribosomal tunnel as a functional environment for nascent polypeptide folding and translational stalling. Curr Opin Struc Biol 21, 274-282.

Woolstenhulme, C.J., Guydosh, N.R., Green, R., and Buskirk, A.R. (2015). Highprecision analysis of translational pausing by ribosome profiling in bacteria lacking EFP. Cell Reports 11, 13-21.

Yanagitani, K., Kimata, Y., Kadokura, H., and Kohno, K. (2011). Translational Pausing Ensures Membrane Targeting and Cytoplasmic Splicing of XBPI $u$ mRNA. Science 331, 586-589. 
Zhao, G., Kong, W., Weatherspoon-Griffin, N., Clark-Curtiss, J., and Shi, Y. (2011). $\mathrm{Mg}^{2+}$ facilitates leader peptide translation to induce riboswitch-mediated transcription termination. EMBO J 30, 1485-1496.

Zylber, E.A., and Penman, S. (1970). The effect of high ionic strength on monomers, polyribosomes, and puromycin-treated polyribosomes. Biochimica et Biophysica Acta 204, 221-229. 


\section{Figure legends}

Figure 1. N-terminal DE/P clusters in $E$. coli ORFs induce IRD.

A. The N-terminal 10 amino acids of the IRD-candidate ORFs are fused to lacZ (Nt10). To evaluate the translation continuity of the DE/P clusters in the ORFs $\left(D E / P^{+}\right), \mathrm{D}, \mathrm{E}$ and $\mathrm{P}$ in the $\mathrm{N}$-terminal regions were replaced with $\mathrm{N}, \mathrm{Q}$ and $\mathrm{A}$, respectively (no $D E P$ ).

B. N-terminal DE/P cluster-dependent translation attenuation in vivo. Each of the Nt10 constructs with or without $\mathrm{DE} / \mathrm{P}$ residues were expressed in $E$. coli, and $\beta$ galactosidase activities were determined (Miller, 1972). The downstream translation frequency corresponding to IRD was calculated as the $\mathrm{LacZ}$ activity ratio $\left[\mathrm{DE} / \mathrm{P}^{+} /\right.$no DEP], termed the translation continuation (T.C.) index.

C. Peptidyl-tRNA accumulation during the $m g t L$ translation. The Nt10 mgtL-lacZ construct and its variant with the DE/P replacement (no DEP) were translated by the PURE system in the presence of ${ }^{35} \mathrm{~S}$-methionine, treated with peptidyl-tRNA hydrolase (Pth) as indicated, and separated by neutral pH SDS-PAGE with an optional RNase A (RN) pretreatment. Peptidyl-tRNAs are indicated by arrows with a schematic label. Radioactive formyl methionyl-tRNA and stop codon-terminated product are indicated as "M" and "CC" (translation-completed chain), respectively.

D. Peptidyl-tRNA accumulation during the translation of other Nt10 constructs.

Figure 2. Exit tunnel-occupying nascent polypeptides minimize the risk of IRD in two different manners.

A. IRD-dependent translation attenuation by $m g t L$ at the early (no insert, upper schematic) or middle ( $\mathrm{FFP}^{+}$, lower schematic) stage of translation elongation in vivo. Translation continuity indices were evaluated as in Figure 1.

B. Schematic of the working hypothesis that a tunnel-occupying preceding nascent polypeptide stabilizes the translating ribosome to prevent IRD.

C. Schematic of the $m g t L-l a c Z$ variants, in which various lengths of the N-terminal portions of GFP or other ORFs were inserted before the $m g t L$ sequence.

D. Inserted amino acid length-dependent IRD-counteraction. The counteracting effects were expressed as the downstream translation continuities $\left[\mathrm{DE} / \mathrm{P}^{+} /\right.$no DEP] of each $m g t L-l a c Z$ variant. The N-terminal regions of E. coli GFP (panel 1), DnaK (panel 2), or DHFR (panel 3) were utilized as the preceding nascent polypeptides. Results of wild type (black line) and IRD-prone $\Delta \mathrm{bL} 31$ (blue line) cells are shown.

E. IRD-counteracting effects of the N-terminal insertions in a reconstituted in vitro translation system (PURE system). The ratio of the completed chain (CC) per aborted 
peptidyl-tRNA (pep-tRNA) accumulated in the PURE system reaction was calculated as the downstream translation continuation of each $m g t L$-lacZ variant.

F. IRD-counteracting effect of short and long nascent polypeptides within the tunnel. Downstream translation continuation of $m g t L-l a c Z$ variants with a 5 aa- or 30 aainsertion ( 5 or 30 aa-EPDP) in the in vivo reporter (left, $n=37$ ) or the in vitro translation assay (right, $n=21$ ) are represented by boxplots.

G. Individual values of in vivo downstream translation continuation of the $m g t L-l a c Z$ variants with the 5 aa- (white dots) or 30 aa-EPDP constructs (black dots).

H. Two-dimensional plots of downstream translation continuation of the 5 aa-EPDP constructs and averaged van der Waals molecular radii of inserted sequences. Both in vivo (left) and in vitro (right) experiments are represented with Spearman's correlation coefficients.

I. Schematic of the ribosomal tunnel occupancy by the bulkiness of the nascent polypeptide adjacent to the PTC.

\section{Figure 3. Effect of mutations in the constriction site within the exit tunnel on the} IRD-counteraction.

A. Structure of the uL4-uL22 constriction site (PDB: 4V5H). Deleted residues in the $\mathrm{uL} 22 \Delta$ loop mutation are indicated in red.

B. Schematic of the working hypothesis that stabilization of the translating ribosome depends on interactions between the ribosomal tunnel and the tunnel-occupying nascent polypeptide.

C. Inserted amino acid length-dependent IRD-counteracting effects in the uL22 2 loopribosome. Messenger RNAs encoding a 5 aa- (left) or 30 aa- (right) insertion and EPDP (used in Figure 2) were individually translated by customized PURE systems including the indicated ribosome variants. Evaluated downstream translation continuation indices of wild type, $\Delta \mathrm{bL} 31$ or uL22 2 loop ribosomes are shown.

D. Preceding nascent polypeptide-length dependency on the IRD counteraction in the ribosome variants. The N-terminal regions of E. coli DnaK (panel 1), GrpE (panel 2) or NuoN (panel 3) were utilized as preceding nascent polypeptides.

E. Structure of the uL23 signaling loop (PDB: 4V5H) and schematic drawing of the tunnel structure. Deleted residues in the uL23 3 loop mutation are indicated in red.

F. Translation continuity of ORFs with the IRD-inducing motif located in the middle of the ORFs. Messenger RNAs encoding rpoD (panel 1), yihI (panel 2) or GFP-10E (panel 3) carrying 10 consecutive $\mathrm{D} / \mathrm{E}$ residues were translated in vitro and the translation continuities were evaluated as described above. 
G. Translation continuity of ORFs with an IRD sequence without a preceding nascent polypeptide occupying the tunnel. Messenger RNAs encoding mgtL (panel 1), FLAG tag (panel 2) or a mixture of 5 aa- EPDP (panel 3) were translated in vitro and analyzed as described above.

Figure 4. Genome-wide biases in amino acids distributions and van der Waals radii.

A. Distributions of the DE/P-rich sequence (more than $3 \mathrm{DE} / \mathrm{P}$ in a 10 residue-moving window) appearance at the $\mathrm{N}$-terminal regions (left) and in the middle (right) of entire E. coli ORFs.

B. Averaged van der Waals molecular radii of every 5 aa-window at the $\mathrm{N}$-terminal regions (left) and in the middle (right) of entire E. coli ORFs.

Figure 5. Ribosome profiling analysis to survey the ribosome occupancy around the N-terminal regions.

A. Schematic representation showing that the ribosome is released from the mRNA by IRD, leading to a decline in ribosome-protected fragments (RPFs) after translation of IRD-inducing sequences.

B. IRD frequency around the N-terminal region was evaluated from the ratio of RPF counts in the $12^{\text {th }}-21^{\text {st }}$ aa to those in the $2^{\text {nd }}-11^{\text {th }}$ aa including DE/P-rich residues.

C. Distribution of the RPFs on the $y b e X$ mRNA in wild type cells. The region of the Nterminal acidic-rich sequence is highlighted.

D. Ratio of RPF counts in the $12^{\text {th }}-21^{\text {st }}$ aa to those in the $2^{\text {nd }}-11^{\text {th }}$ aa. The data are classified as follows: Total: all dataset (a), E. coli ORFs with DE/P-rich and bulky (b) or small (c) radii-residues in $\mathrm{N}$-terminal regions. A detailed representation of the classification is shown in Supplementary Figure S5B. The data from two biological replicates of wild type (panel 1) or IRD-prone $\Delta$ bL31 (panel 2) cells are shown. Schematics at the bottom represent the nascent chain occupying the tunnel during the translation of the $2^{\text {nd }}-11^{\text {th }}$ aa.

E. Ratio of RPF counts in 10 amino acids with "small" and DE/P-rich residues to those in the following 10 amino acids at different stages of elongation. RPF counts for 10 amino acids with "small" and DE/P-rich residues were summed from the most Nterminal $\left(2^{\text {nd }}-11^{\text {th }},(a)\right)$ to the subsequent every 10 amino acids windows, (b)-(d). RPF counts for 10 amino acids with "small" and DE/P-rich residues in (e) were summed from $\mathrm{X}-30^{\text {th }}$ to $\mathrm{X}-21^{\text {st }}$, where $\mathrm{X}$ represents the last amino acid in the ORFs. The data from two biological replicates of wild type (panel 1) or IRD-prone $\Delta$ bL31 (panel 2) cells are shown. The corresponding data for "bulky" and DE/P-rich residues are 
shown in Supplementary Figure S5C.

Figure 6. Exit tunnel-occupying nascent polypeptide physically stabilizes translating $70 \mathrm{~S}$ ribosome as an inter-subunit bridge.

A. Stability of the translating ribosome complex with or without the bL31 protein. Cellular extracts of wild type (panel 1) or $\Delta$ bL31 (panel 2) cells were fractionated by sucrose density gradient ultracentrifugation (SDG) in the presence of $10 \mathrm{mM}$ magnesium ion (no treatment, black line). Prior to the extraction, E. coli cells were treated with puromycin $(\mathrm{Pm}$, blue line $)$ or chloramphenicol $(\mathrm{Cm}$, red line $)$ to remove or keep the nascent polypeptide within the ribosome, respectively. Distributions of the ribosomes were monitored by $\mathrm{A}_{254}$ measurements. Fractions including the $70 \mathrm{~S}$ ribosome are expanded for comparison.

B. Experimental design to evaluate the stability of the translating ribosomes. MNase: micrococcal nuclease.

C. Stability of the $70 \mathrm{~S}$ ribosome complex with or without a tunnel-occupying nascent polypeptide in wild type (panels 1 and 3 ) or $\Delta$ bL31 (panels 2 and 4) cells. Pooled 70S translating ribosomes with (panels 3 and 4) or without (panels 1 and 2) the puromycin treatment were fractionated by SDG in the presence of $10 \mathrm{mM}$ magnesium ion. Recorded $\mathrm{A}_{254}$ values are shown. The peaks indicated as the asterisk (*) in panel 1 and 3 seem to be an aberrant form of the 70S ribosome due to the MNase treatment. 
A

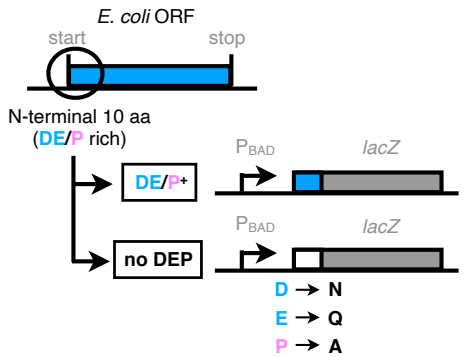

B

C

DE/P + no DEP treatment - PthRN - PthRN

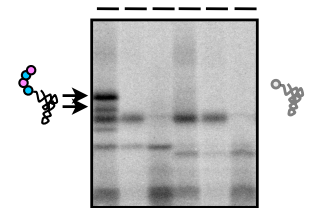


A
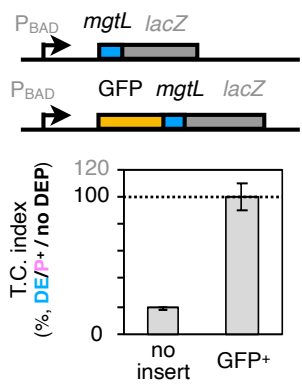

D
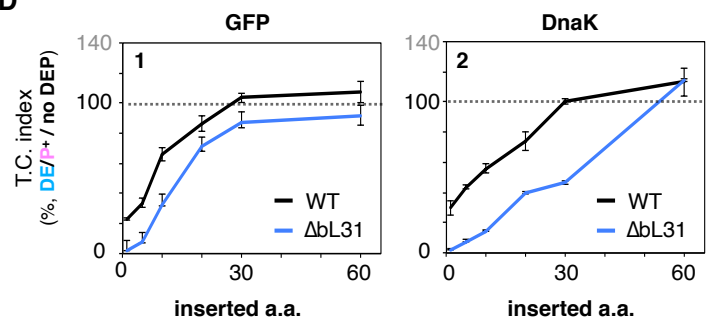

B

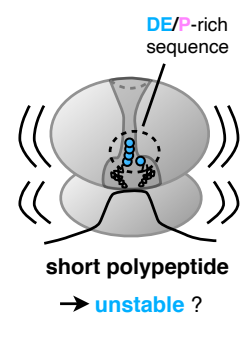

C

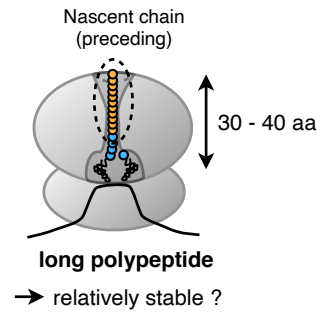

$\mathbf{F}$

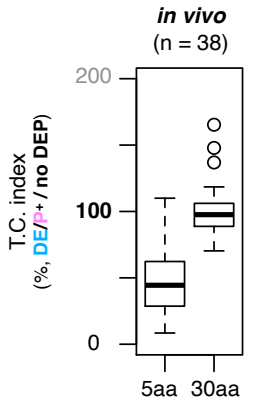

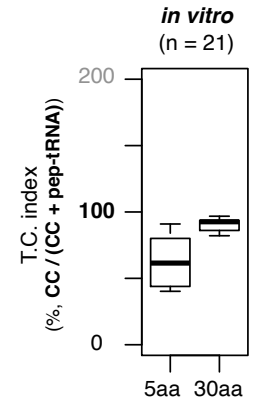

G

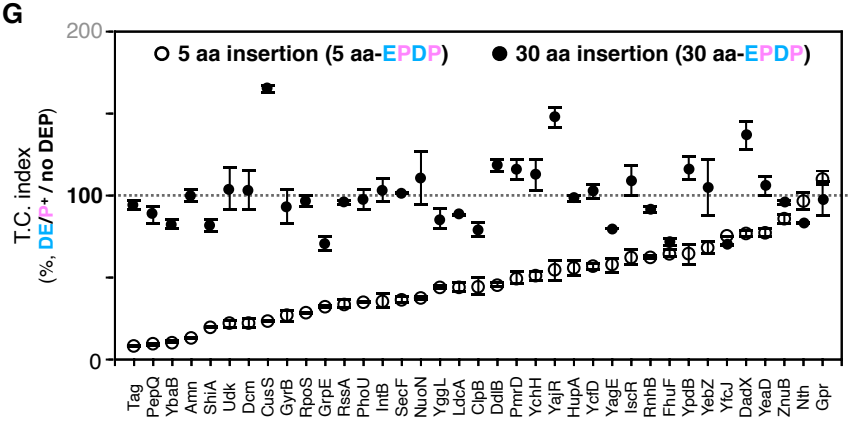

H

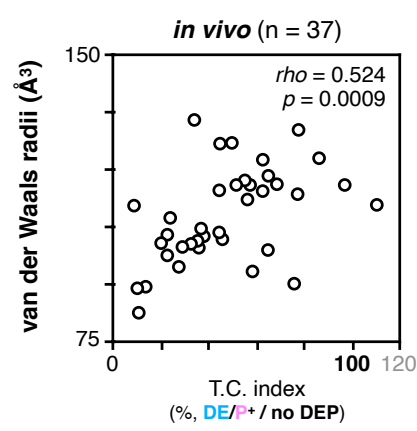

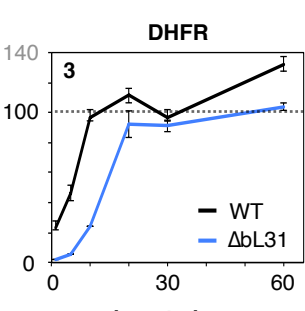

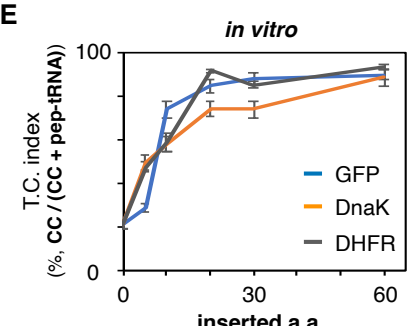

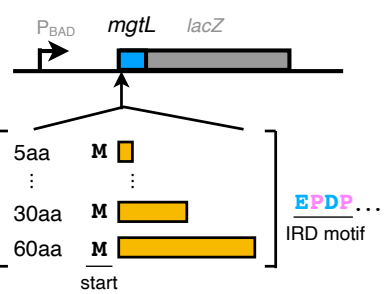

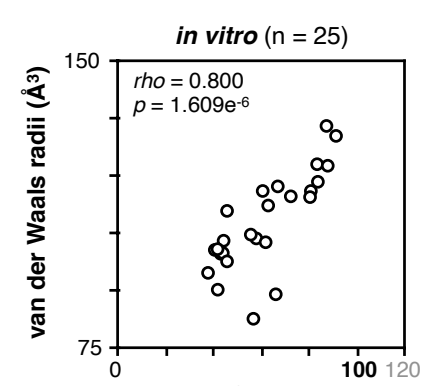

T.C. index

(\%, CC / (CC + pep-tRNA))
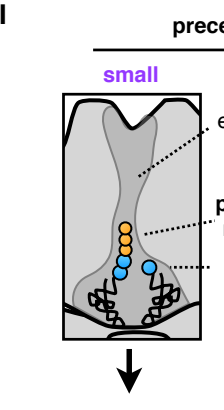

unstable

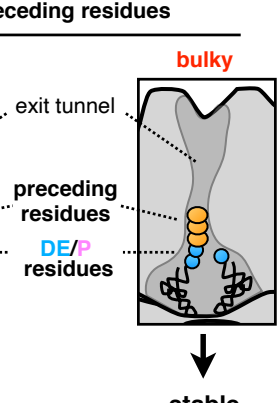

stable 
A

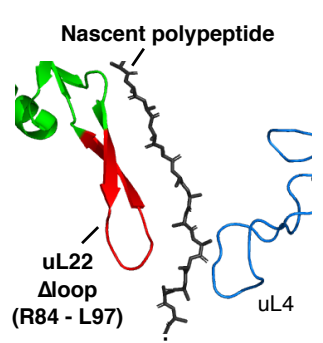

PTC
B

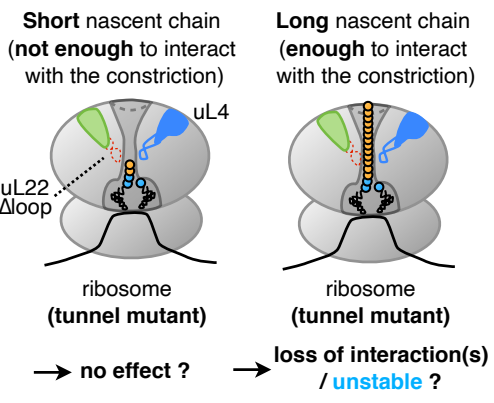

C

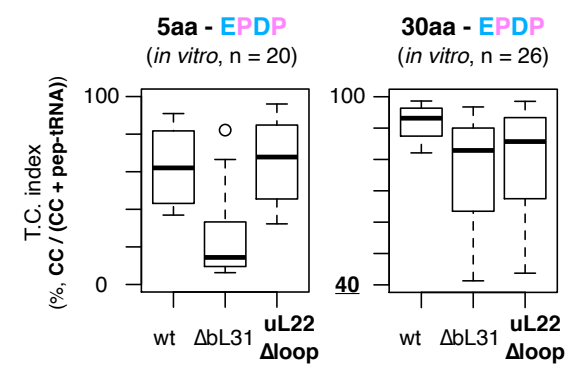

D

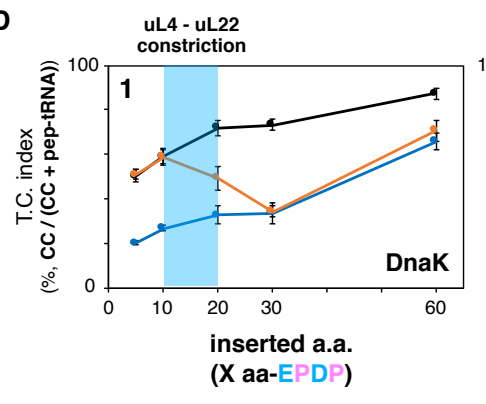

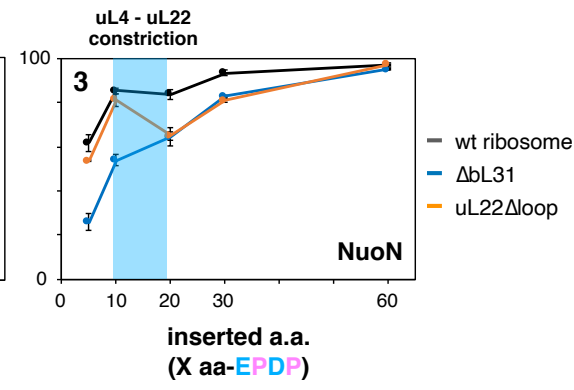

E

$\mathbf{F}$

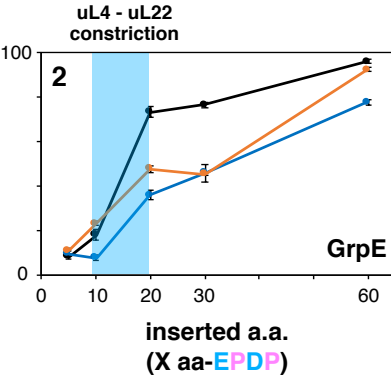

Yihl

GFP-10E
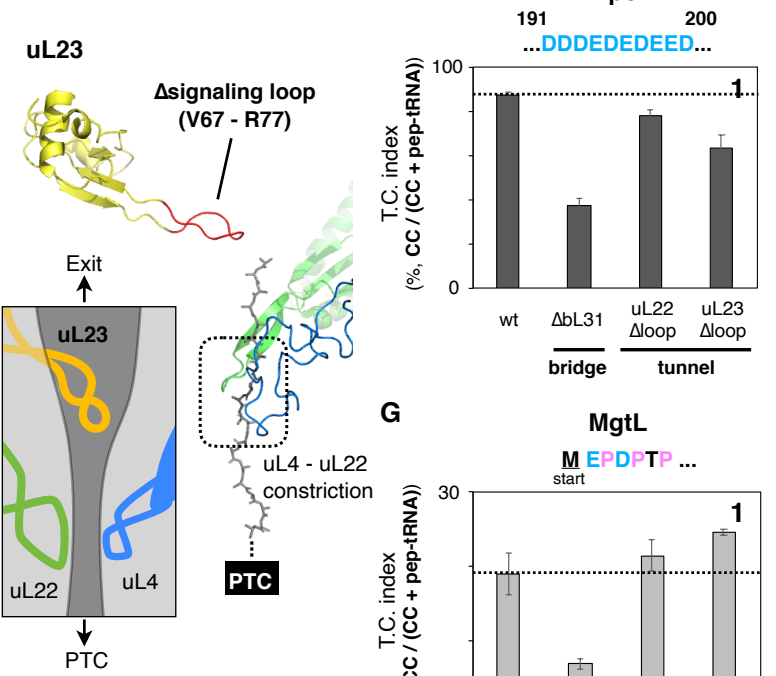

$147 \quad 156$

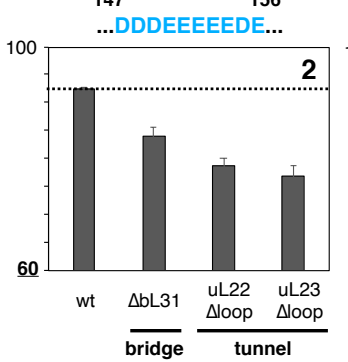

$379 \quad 388$
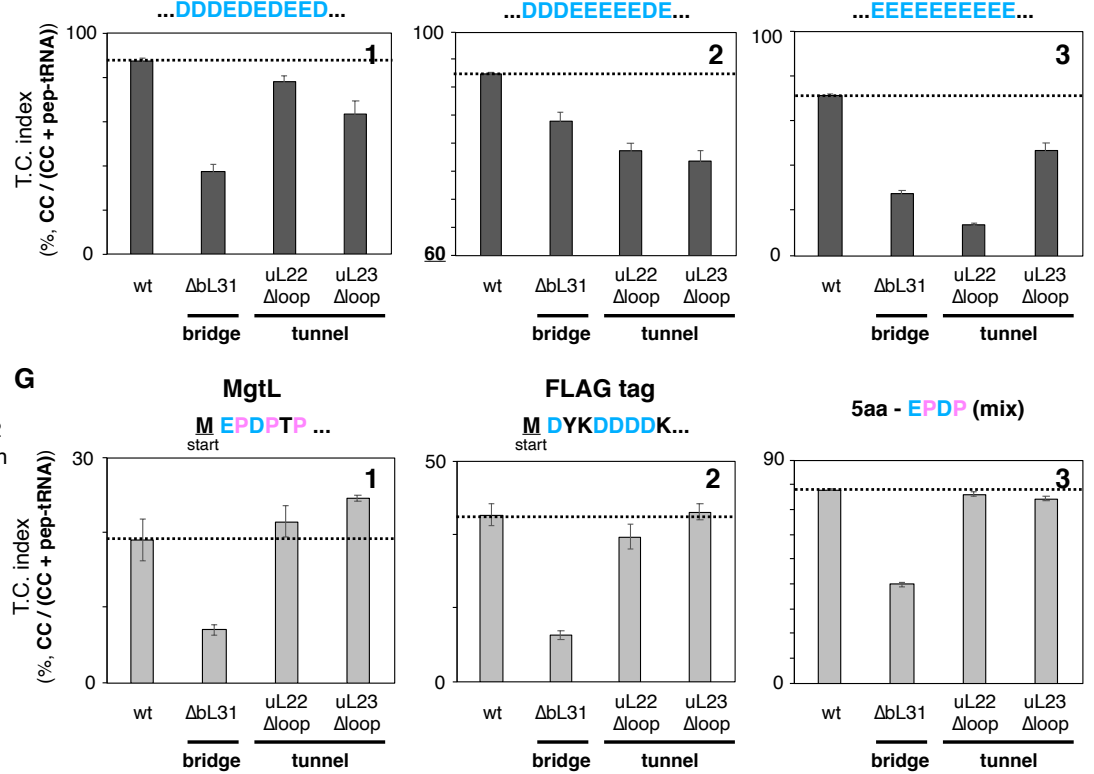

5aa - EPDP (mix)

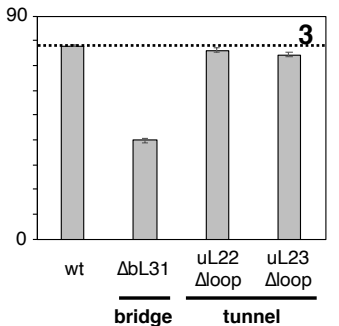


A
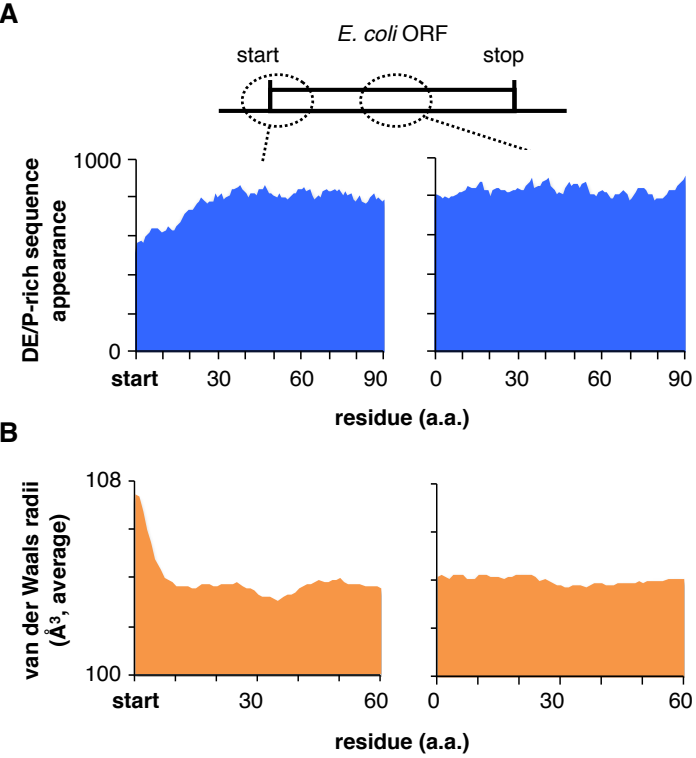

Figure 4 
A

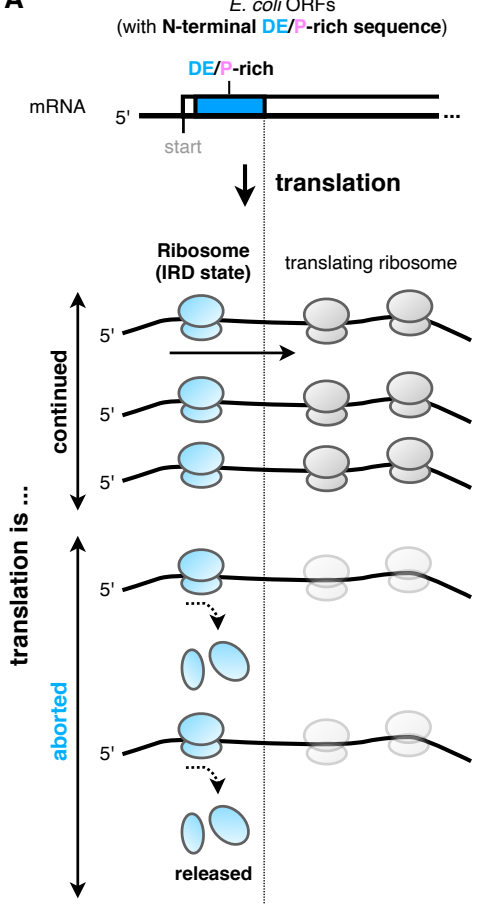

B

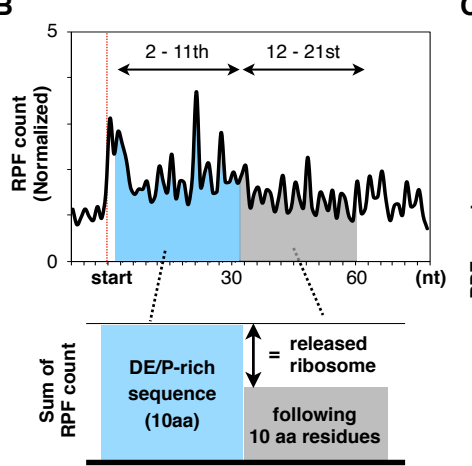

2nd 11th following 10 aa

(a)
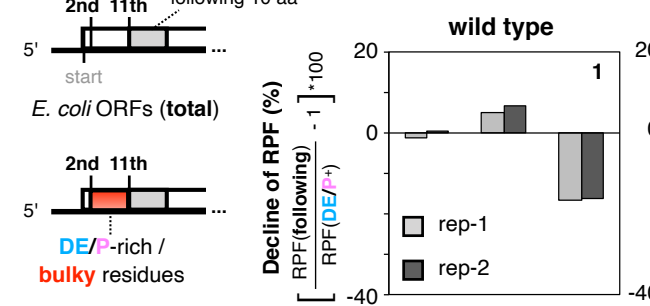

(b) 5

E$$
\text { 2nd 11th }
$$

(c) (a) (b) (c)

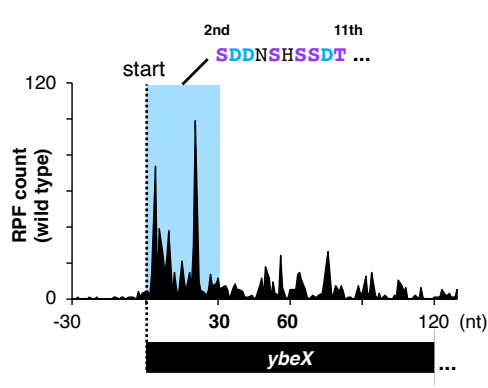

C

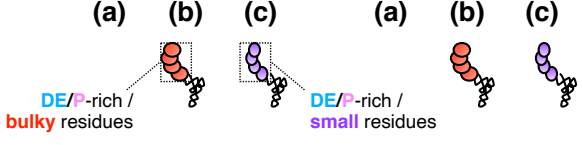
coli ORFs (with DE/P-rich / small residues at ...
(a)
2nd 11th

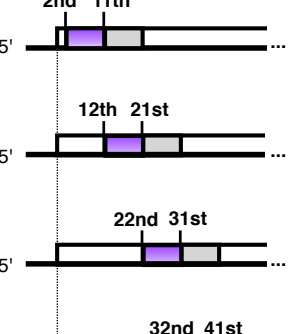
(d)

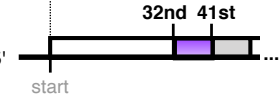
:
(e)
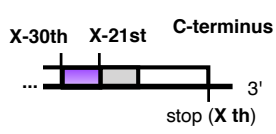
DE/P-rich / stop $(\mathbf{X}$ th)
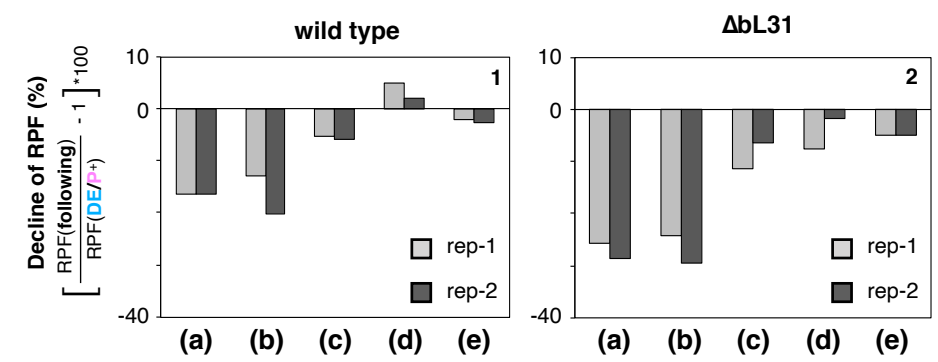
Nascent chain within $>1$ (a) $>10>20$

$\begin{array}{lllll}\text { (a) } & \text { (b) } & \text { (c) } & \text { (d) } & \text { (e) }\end{array}$

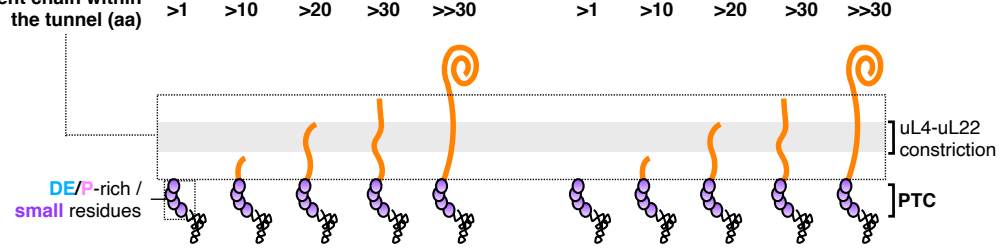


A

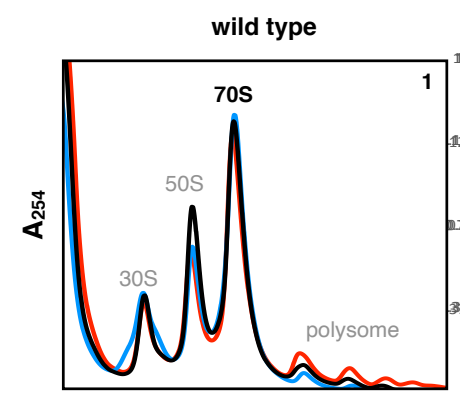

$\Delta \mathrm{bL31}$

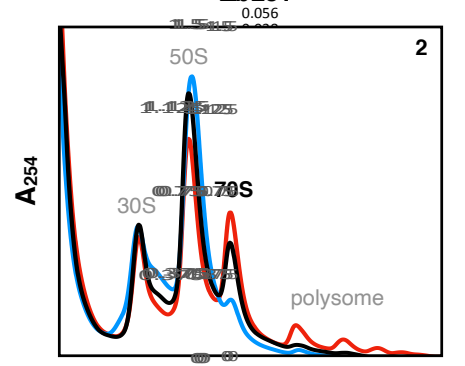

$70 \mathrm{~S} 70 \mathrm{~S}$

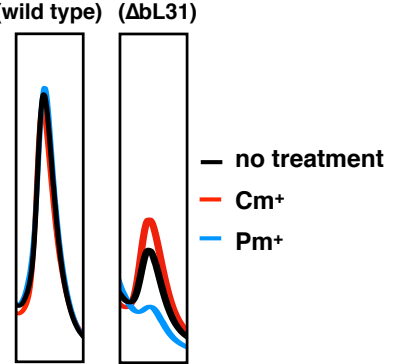

B

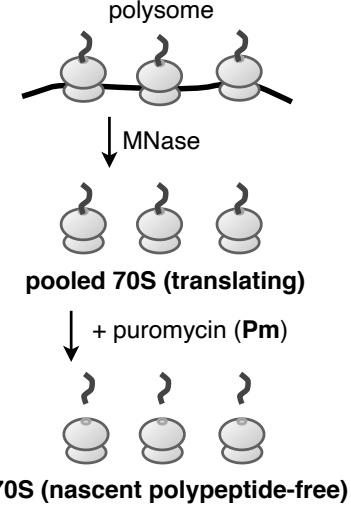

C

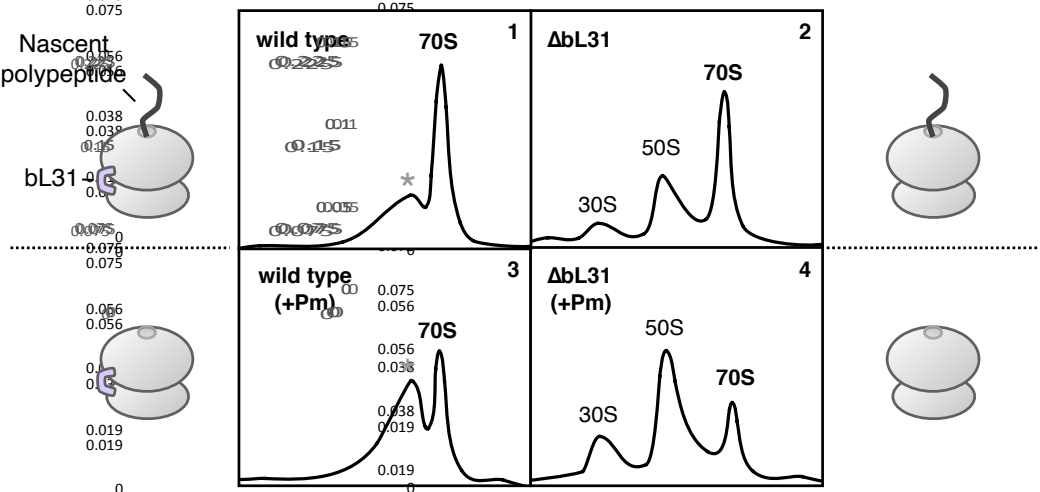




\section{STAR Methods}

\section{CONTACT FOR REAGENT AND RESOURCE SHARING}

Please direct any requests for further information or reagents to the lead contact, Hideki Taguchi (taguchi@bio.titech.ac.jp).

\section{EXPERIMENTAL MODEL AND SUBJECT DETAILS}

\section{E. coli strains, plasmids, and primers}

E. coli strains, plasmids and oligonucleotides used in this study are listed in Table S1. The ASKA plasmid library (Kitagawa et al., 2006) were obtained from National Institute of Genetics (NIG). Plasmids were constructed using standard cloning procedures and Gibson assembly. Detailed schemes were summarized in Table S1, and sequences of constructed plasmids will be uploaded to the Mendeley repository.

PCR-amplified DNA fragment from pDGT1 using primers PR0058 and PR0059 was electroporated into strain BW25113 harboring pKD46 (Datsenko and Wanner, 2000) and pCY927 (rpsJ-rps $Q$ operon), selecting for a trimethoprim (20 $\mu \mathrm{g} / \mathrm{ml})$-resistant transformant. Genome-introduced $\Delta$ rpsJ-rpsQ ::FRT-DHFR-FRT mutation was transferred by Phage P1 into MG1655 harboring pCY873 (rpsJ-rps $Q$ operon), pCY920 (rpsJ-rps $Q$ operon with uL22 $\Delta$ loop mutation), or pCY1457 (rpsJ-rps $Q$ operon with $\mathrm{uL} 23 \Delta$ loop mutation), respectively.

\section{METHODS DETAILS}

\section{In vitro translation and product analysis}

A coupled transcription-translation reaction was performed using PUREfrex 1.0 (GeneFrontier) in the presence of ${ }^{35} \mathrm{~S}$-methionine at $37^{\circ} \mathrm{C}$ for $30 \mathrm{~min}$. DNA templates were prepared by PCR, as summarized in Table S2. Mutant ribosomes ( $\Delta$ bL31, uL22 2 loop, uL23 $\Delta$ loop) purified as described (Ohashi et al., 2007) were used when indicated. After $30 \mathrm{~min}$ incubation, the reaction mixture was treated with $200 \mu \mathrm{g} / \mathrm{ml}$ of puromycin for $5 \mathrm{~min}$ at $37^{\circ} \mathrm{C}$. Half of the mixture was further treated with $1 \mu \mathrm{M}$ of purified Pth for $10 \mathrm{~min}$ at $37^{\circ} \mathrm{C}$ when indicated. The reaction was stopped by dilution into an excessive volume of 5\% TCA. After standing on ice for 10 min or more, samples were centrifuged for $3 \mathrm{~min}$ at $4{ }^{\circ} \mathrm{C}$, and the supernatant was discarded by aspiration. Precipitates were then vortexed with $0.9 \mathrm{ml}$ of acetone, centrifuged again, and dissolved in SDS sample buffer (62.5 mM Tris-HCl pH 6.8, 2\% SDS, 10\% glycerol, $50 \mathrm{mM} \mathrm{DTT})$ that had been treated with RNasecure (Ambion). Finally, the sample without Pth treatment was 
divided into two portions, one of which was incubated with $50 \mu \mathrm{g} / \mathrm{ml}$ of RNase A (Roche) at $37^{\circ} \mathrm{C}$ for $30 \mathrm{~min}$ and separated by WIDE range SDS-PAGE system (Nakalai tesque).

\section{Translation continuation (TC) index in vitro}

The ratio of the translation-completed chain (CC) against Pth-sensitive polypeptidyltRNAs (pep-tRNA), which signifies the occurrence of IRD-induced abortion in the total in vitro translation products, was calculated as translation continuation (TC) index. In detail, the radioactivity ( ${ }^{35} \mathrm{~S}$-methionine) proportion of "CC" and "pep-tRNA" among the sample with neither Pth- nor RNase-treatment were quantified by Multi Gauge (Fujifilm), and the TC value (in \%) is obtained by the following formula.

$\mathrm{TC}$ index $($ in vitro $)=(\mathrm{CC}) /[(\mathrm{CC})+($ pep-tRNA $)]$

The average of three independent experiments was presented as TC indices, except for Figure 3C (Figure S3).

\section{$\beta$-galactosidase assay}

\section{TC index in vivo}

E. coli cells harboring lacZ reporter plasmid were grown overnight at $37^{\circ} \mathrm{C}$ in $\mathrm{LB}$ medium supplemented $100 \mu \mathrm{g} / \mathrm{ml}$ ampicillin. They were inoculated into fresh LB medium containing $2 \times 10^{-4} \%$ arabinose and $100 \mu \mathrm{g} / \mathrm{ml}$ ampicillin and were grown at $37^{\circ} \mathrm{C}$ for $\sim 2.5 \mathrm{hrs}\left(\mathrm{A}_{660}=\sim 0.4\right)$. Then $20 \mu \mathrm{l}$ portion was subjected to $\beta$-galactosidase assay as described (Miller, 1972). Estimated miller unit (m.u.) were subjected to the following formula to calculate the TC index (in vivo).

$\mathrm{TC}$ index $($ in vivo $)=m . u .\left(\mathrm{DE} / \mathrm{P}^{+}\right) / m . u .($ no DEP $)$

TC indices were calculated from three independent experiments, and plasmids used in each experiment were summarized in Table S2.

\section{$\mathrm{Mg}^{2+}$-depletion}

E. coli cells harboring $l a c Z$ reporter plasmid were grown overnight at $37^{\circ} \mathrm{C}$ in $\mathrm{M} 9$ medium (Miller, 1972) supplemented with $0.1 \%$ casamino acids, $0.1 \mathrm{mM} \mathrm{CaCl}_{2}, 50 \mu \mathrm{M} \mathrm{MgSO}_{4}$, $50 \mu \mathrm{g} / \mathrm{ml}$ of thiamine, $0.4 \%$ glycerol and $100 \mu \mathrm{g} / \mathrm{ml}$ ampicillin. They were inoculated into fresh M9 medium containing $2 \times 10^{-4} \%$ arabinose, and were grown at $37^{\circ} \mathrm{C}$ for $4 \mathrm{hrs}$. Then, $20 \mu$ portion was subjected to $\beta$-galactosidase assay as described (Miller, 1972). 
The concentration of $\mathrm{MgSO}_{4}$ in $\mathrm{M} 9$ medium was adjusted to $10 \mathrm{mM}$ or $10 \mu \mathrm{M}$ when indicated.

\section{Amino acids distribution analysis}

We calculated the number of ORFs with more than three D,E and $\mathrm{P}$ residues within 10amino acids moving windows in both $\mathrm{N}$-terminal and internal regions in entire $E$. coli ORFs. Datasets of protein sequences were obtained from UniProt (https://www.uniprot.org).

\section{Ribosome profiling}

\section{a. Sequencing of ribosome-protected fragment (RPF)}

E. coli MG1655 (wild type) or ECY0228 (MG1655 $\Delta$ bL31) strain was grown overnight in $\mathrm{LB}$ medium at $37^{\circ} \mathrm{C}$. They were inoculated into fresh LB medium and were grown at $37^{\circ} \mathrm{C}$. When $\mathrm{A}_{660}$ reached to 0.5 , cells were treated with $100 \mu \mathrm{g} / \mathrm{ml}$ of chloramphenicol and collected by filtration ( $0.45 \mu \mathrm{m}$ MF membrane). Filter-trapped E. coli cells were scraped and dropped into liquid nitrogen dispensed to $50 \mathrm{ml}$ corning tube in advance. In addition, RP buffer (20 mM Tris- $\mathrm{HCl} \mathrm{pH} 7.5,150 \mathrm{mM} \mathrm{NH} 4 \mathrm{Cl}, 10 \mathrm{mM} \mathrm{MgCl} 2,1 \mathrm{mM}$ DTT, $5 \mathrm{mM} \mathrm{CaCl} 2,1 \%$ Triton X-100, $100 \mu \mathrm{g} / \mathrm{ml}$ chloramphenicol) was added to form frozen droplets. The cell droplets were disrupted by Multi-Beads Shocker [MB601U(S), Yasui Kikai], thawed and treated with 25 unit/ $\mu$ of Turbo ${ }^{\mathrm{TM}}$ DNase (Thermo Fisher Scientific) for $10 \mathrm{~min}$ on ice. After centrifugation at $20,000 \mathrm{~g}$ for $10 \mathrm{~min}$ at $4{ }^{\circ} \mathrm{C}$, the supernatant was collected and $35 \mu \mathrm{g}$ RNA per sample was treated with 150 unit of Micrococcal Nuclease (MNase, Roche) for $45 \mathrm{~min}$ at $25^{\circ} \mathrm{C}$. RNA fragments ranging from 17 to $50 \mathrm{nt}$ were gel-excised as ribosome-protected fragment (RPF). Following library preparation was performed as previously described (McGlincy and Ingolia, 2017). A Ribo-Zero rRNA Removal Kit for Bacteria (Illumina) was used to deplete rRNA contamination. The libraries were sequenced on a HiSeq4000 (Illumina). After depleting the reads originating from noncoding RNAs, the remaining reads were mapped to the $E$. coli genome sequence (NC_000913.2). Empirically, we defined an A-site position, which is essentially the same as the $3^{\prime}$ assignment as described previously (Woolstenhulme et al., 2015).

\section{b. Classification of $E$. coli ORFs by sequential features} "DE/P-rich" sequence

We defined two or more $\mathrm{D}, \mathrm{E}$ or $\mathrm{P}$ residues within continuous three codons as " $\mathrm{DE} / \mathrm{P}^{+}$ cluster". Furthermore, two or more "DE/ $\mathrm{P}^{+}$clusters" within the continuous ten codons 
were defined as "DE/P-rich" sequences that potentially induce IRD and utilized for classification of $E$. coli ORFs.

"Van der Waals radii"

The averaged van der Waals radii of ten residues belonging to "DE/P-rich" sequence at indicated region was calculated and utilized for classification of $E$. coli ORFs as follows. "DE/P-rich sequence" with bulky-sized residues: $40 \%$ of the larger one "DE/P-rich sequence" with small-sized residues: $40 \%$ of the smaller one

\section{c. Calculation of normalized RPF}

We utilized house-made script to describe the distribution of normalized RPF counts among the subsets of $E$. coli ORFs classified as described above. Briefly, the relative RPF count in each nucleotide position was calculated for each ORF at first. Then, the obtained relative RPF count in each position was averaged for each subset. We excluded the ORFs with less than 150 bp length, poor expression (less than 400 RPF count/gene) or concentrated RPF count at specific site (b0621, b2094, b2218, b3709 and b2744) to convince the statistical analyses. The sum of normalized RPF counts among the indicated region was compared to that of following 10 amino acids residues to estimate the frequency of IRD-dependent translation abortion.

\section{Polysome profile}

E. coli cells were grown in LB medium overnight at $37^{\circ} \mathrm{C}$. They were inoculated into fresh LB medium and were grown at $37^{\circ} \mathrm{C}$. When $\mathrm{A}_{660}$ reaches to 0.5 , cells were optionally treated with $20 \mu \mathrm{g} / \mathrm{ml}$ puromycin for $10 \mathrm{~min}$ or $200 \mu \mathrm{g} / \mathrm{ml}$ chloramphenicol for $1 \mathrm{~min}$. Cell culture was mixed with crushed ice and centrifuged at 8,000 rpm for $3 \mathrm{~min}$ at $4{ }^{\circ} \mathrm{C}$. Pelleted cells were resuspended by polysome buffer $[20 \mathrm{mM}$ Tris-acetate $\mathrm{pH} 7.5$, $150 \mathrm{mM} \mathrm{NH}_{4} \mathrm{Cl}, 10 \mathrm{mM} \mathrm{Mg}(\mathrm{OAc})_{2}, 1 \mathrm{mM}$ DTT, $(200 \mu \mathrm{g} / \mathrm{ml}$ chloramphenicol was added when indicated)] and dropped into liquid nitrogen dispensed to $50 \mathrm{ml}$ corning tube in advance. Frozen cell droplets were disrupted by Multi-Beads Shocker [MB601U(S), Yasui Kikai] and treated with 10 unit of Turbo ${ }^{\mathrm{TM}}$ DNase (Thermo Fisher Scientific) for $20 \mathrm{~min}$ on ice. Lysate was centrifuged at $20,000 \mathrm{~g}$ for $10 \mathrm{~min}$ at $4^{\circ} \mathrm{C}$. The supernatant was layered onto the top of 10-30\% sucrose gradient containing the polysome buffer in Open Top Polyclear ${ }^{\mathrm{TM}}$ Centrifuge Tubes (14 x $89 \mathrm{~mm}$, SETON) and centrifuged at 39,000 rpm for $4 \mathrm{~h}$ at $4{ }^{\circ} \mathrm{C}$ (Beckman OptimaL-90K, SW41-Ti), followed by fractionation using Gradient Station (BIOCOMP) equipped with MICRO COLLECTOR AC-5700 (ATTO). 
Distribution of ribosome was monitored by $\mathrm{A}_{254}$ measurements using $\mathrm{BIO}$ MINI UV MONITOR (AC-5200S, ATTO).

\section{Stability assay for $70 \mathrm{~S}$ translating ribosome complex}

E. coli cells were grown in LB medium overnight at $37^{\circ} \mathrm{C}$. They were inoculated into 2x YTPG medium supplemented with Antifoam SI (Fujifilm-WAKO) till A660 reaches to 3.0. Cells were collected and extracted as described in polysome profile analysis without antibiotic treatment. Prepared cell extract was fractionated by $10-50 \%$ sucrose gradient ultracentrifugation at 39,000 rpm for $2.5 \mathrm{~h}$ at $4^{\circ} \mathrm{C}$ (Beckman OptimaL-90K, SW41-Ti). The fractions containing polysome (larger than the $70 \mathrm{~S}$ monosome) were collected and again centrifuged at 50,000 rpm for $3 \mathrm{~h}$ at $4^{\circ} \mathrm{C}$ (Beckmann Optima ${ }^{\mathrm{TM}} \mathrm{TLX}$ Ultracentrifuge, TLA100.3) to pellet down the polysome particles. The pellet was once rinsed by the polysome buffer and resolved by overnight-standing within the polysome buffer supplemented with $5 \mathrm{mM} \mathrm{CaCl}_{2}$ at $4{ }^{\circ} \mathrm{C}$. Fifty $\mu$ l of prepared polysome $\left(\mathrm{A}_{260}=\sim 8.0\right)$ was treated with $100 \mu \mathrm{g} / \mathrm{ml}$ of puromycin for $1 \mathrm{~h}$ at room temperature $\left(24^{\circ} \mathrm{C}\right)$ with gentle mixing in the presence of 300 unit of Micrococcal Nuclease (MNase, Roche). Reaction mixture was mixed with $100 \mu$ of polysome buffer and layered onto the top of $10-30 \%$ sucrose gradient containing the polysome buffer in Open Top Polyclear ${ }^{\mathrm{TM}}$ Centrifuge Tubes (14 x $89 \mathrm{~mm}, \mathrm{SETON})$ and centrifuged at 39,000 rpm for $3 \mathrm{~h}$ at $4^{\circ} \mathrm{C}($ Beckman OptimaL-90K, SW41-Ti) to record $\mathrm{A}_{254}$ as described above.

\section{Data analyses}

Statistical analyses were conducted by using the software R (https://www.rproject.org). 